\title{
G

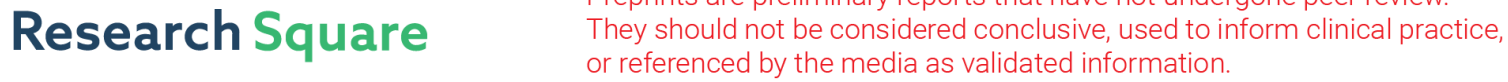 \\ Selective activation of cannabinoid receptor-2 reduces white matter injury via PERK signaling in a rat model of traumatic brain injury
}

\section{li jiang ( $\nabla$ drjiangli2019@163.com )}

Chongqing University of Medical Science Clinical College: The First Affiliated Hospital of Chongqing Medical University

\section{Lin Li}

Chongqing University of Medical Science Clinical College: The First Affiliated Hospital of Chongqing Medical University

\section{Qing Luo}

department of ultrasound nan Chong central hospital

\section{Bin Shang}

department of neurosurgery nan Chong central hospital

\section{Xiaomin Yang}

Chongqing University of Medical Science Clinical College: The First Affiliated Hospital of Chongqing Medical University

Jiru Zhou

Chongqing University of Medical Science Clinical College: The First Affiliated Hospital of Chongqing Medical University

\section{Qiuling Pan}

Chongqing University of Medical Science Clinical College: The First Affiliated Hospital of Chongqing Medical University

\section{$\mathrm{Na} \mathrm{Wu}$}

Chongqing University of Medical Science Clinical College: The First Affiliated Hospital of Chongqing Medical University

\section{Wei Tang}

Chongqing University of Medical Science Clinical College: The First Affiliated Hospital of Chongqing Medical University

\section{Donglin Du}

Chongqing University of Medical Science Clinical College: The First Affiliated Hospital of Chongqing Medical University

\section{Xiaochuan Sun}

Chongqing University of Medical Science Clinical College: The First Affiliated Hospital of Chongqing Medical University 


\section{Research Article}

Keywords: Traumatic brain injury, Cannabinoid receptor-2, white matter injury, Endoplasmic reticulum (ER) stress, Microglia/macrophage polarization, oligodendrocytes

Posted Date: April 30th, 2021

DOI: https://doi.org/10.21203/rs.3.rs-286920/v2

License: (c) (i) This work is licensed under a Creative Commons Attribution 4.0 International License.

Read Full License 


\section{Abstract}

Background and Purpose: Traumatic brain injury (TBI) destroys white matter, and this destruction is aggravated by secondary neuroinflammatory reactions. Although white matter injury (WMI) is strongly correlated with poor neurological function, understanding of white matter integrity maintenance is limited, and no available therapies can effectively protect white matter. One candidate approach that may fulfill this goal is cannabinoid receptor 2 (CB2) agonist treatment. Here, we confirmed that a selective CB2 agonist, JWH133, protected white matter after TBI.

Methods: The controlled cortical impact (CCI) was used to establish a moderate TBI model in adult male Sprague-Dawley rats (8-10 weeks, 250-300g). The motor evoked potentials (MEPs), open field test, and Morris water maze test were used to assess neurobehavioral outcomes. Brain tissue loss, WM damage, Endoplasmic reticulum stress (ER stress), and microglia responses were evaluated after TBI. The functional integrity of WM was measured by diffusion tensor imaging (DTI) and transmission electron microscopy (TEM). Primary microglia and oligodendrocyte cocultures were used for additional mechanistic studies.

Results: JWH133 increased myelin basic protein (MBP) and neurofilament heavy chain (NF200) levels and anatomic preservation of myelinated axons revealed by DTI and TEM. JWH133 also increased the numbers of oligodendrocyte precursor cells and mature oligodendrocytes. Furthermore, JWH133 drove microglial polarization toward the protective M2 phenotype and modulated the redistribution of microglia in the striatum. Further investigation of the underlying mechanism revealed that JWH133 downregulated phosphorylation of the protein kinase $\mathrm{R}$ (PKR)-like endoplasmic reticulum (ER) kinase (PERK) signaling pathway and its downstream signals eukaryotic translation initiation factor 2 a (elF2a), activating transcription factor 4 (ATF4) and Growth arrest and DNA damage-inducible protein (GADD34); this downregulation was followed by $\mathrm{p}$-Protein kinase $\mathrm{B}$ (p-Akt) upregulation. In primary cocultures of microglia and oligodendrocytes, JWH133 decreased phosphorylated PERK expression in microglia stimulated with tunicamycin and facilitated oligodendrocyte survival. These data reveal that JWH133ultimately alleviates WMI and improves neurological behavior following TBI. However, these effects were were prevented by SR144528(i.p. injection 3 min before receiving JWH133 intraperitoneally), a selective CB2 antagonist.

Conclusions: This work illustrates the PERK-mediated interaction between microglia and oligodendrocytes. In addition, the results are consistent with recent findings that microglial polarization switching accelerates WMI, highlighting a previously unexplored role for CB2 agonists. Thus, CB2 agonists are potential therapeutic agents for TBI and other neurological conditions involving white matter destruction.

\section{Introduction}


More than 50 million people experience traumatic brain injury (TBI) each year worldwide, and approximately half of the world population is likely to have one or more TBIs during their lifetime[1]. Additionally, China has more TBI patients than most other countries (China had more than 1.39 billion TBI patients at the end of 2017, representing approximately $18 \%$ of the world population), making this condition a serious public health concern and placing very large burdens on society and families[1, 2].

TBI causes not only gray matter damage but also severe white matter injury (WMI), thereby disrupting signal transmission and eliciting poor sensorimotor and cognitive functional outcomes[3, 4]. Indeed, the extent of WMI in TBI patients is strongly correlated with neurological deficits, and diffusion tensor imaging (DTI) of white matter presents prognostic value for neurological status[4, 5]. However, there are currently no satisfactory therapeutic strategies to alleviate WMI resulting from TBI. Unfortunately, many preclinical TBI studies have strongly emphasized the protection of gray matter over white matter, which has inevitably contributed to many disappointing results in preclinical trials to date[6].

The endocannabinoid system contains cannabinoid receptors 1 and 2 (CB1 and CB2); two endocannabinoids, anandamide (AEA) and 2-arachidonoylglycerol (2-AG); and enzymes for endocannabinoid anabolism and catabolism[7]. Endocannabinoid signaling is involved in the regulation of brain development, neurotransmitter release, synaptic plasticity, and cytokine release from microglia and is therefore implicated in multiple neurological disorders, such as TBI, stroke, and multiple sclerosis[8]. Furthermore, endocannabinoid signaling in plasma and brain samples is altered in experimental models of neurological disorders. Abundant evidence has confirmed that targeting the endocannabinoid system has medical benefits, which has initiated efforts to develop cannabinoid-based therapeutics. However, these efforts have been disappointing, especially in relation to the unwanted central side effects of CB1 activation, which have limited further development of drugs targeting this receptor. However, the discovery of CB2 and its endogenous cannabinoid receptor ligands has raised new possibilities for safe targeting of the endocannabinoid system[7].

Endoplasmic reticulum (ER) stress and abnormal protein accumulation have been observed in the pericontusional brain and are considered to be significant mechanisms of secondary injury after TBI[9]. Recent studies have shown that activation of protein kinase R (PKR)-like ER kinase (PERK) mediates the unfolded protein response (UPR) signaling pathway that predominates following TBI. Notably, suppressing aberrant activation of PERK relieves brain injury after TBI[10]. However, the influence of CB2 agonism on the TBI-induced activation of PERK is not clear.

Previous studies have verified the anti-inflammatory effects of CB2 activation[11, 12]. Thus, we explored the effects of CB2 activation on microglia/macrophage phenotype polarization and on oligodendrocytes (OLs). We also investigated the interaction mechanism between microglia and OLs. We found that the selective CB2 agonist JWH133 suppresses the PERK signaling pathway, which then serves to inactivate eukaryotic translation initiation factor 2 a (elF2a) via dephosphorylation. elF2a inactivation downregulates the expression of activating transcription factor 4 (ATF4) and GADD34. As a result, Akt 
phosphorylation is upregulated and drives microglia/macrophage polarization toward the M2 phenotype, which helps to protect OLs and alleviate WMI after TBI.

\section{Materials And Methods}

\section{Controlled cortical impact (CCl) model establishment and grouping}

Adult male Sprague-Dawley rats (8-10 weeks, $250-300 \mathrm{~g}$ ) were purchased from the Experimental Animal Center of Chongqing Medical University (Chongqing, China). Rats were generally anesthetized via intraperitoneal (i.p.) injection of chloral hydrate $(3.5 \%, 350 \mathrm{mg} / \mathrm{kg})$, and a circular craniotomy $(5 \mathrm{~mm}$ in diameter) was performed (located $4 \mathrm{~mm}$ posterior to bregma and $2.5 \mathrm{~mm}$ lateral to the sagittal suture over the right parietal cortex). Following the craniotomy, a CCI model was established with a TBI-0310 TBI model system (Precision Systems and Instrumentation, USA) and the following impact parameters, as previously reported: a velocity of $3.5 \mathrm{~m} / \mathrm{s}$, a depth of $2.5 \mathrm{~mm}$, and a dwell time of $200 \mathrm{~ms}$ [13]. The sham rats underwent the same procedures as the $\mathrm{CCl}$ rats but without impact.

The rats were randomly divided into four groups in this study: the sham group, the TBI + vehicle (vehicle) group, the TBI + JWH133 (JWH) group, and the TBI + SR144528 + JWH133 (SR+JWH) group. The sham group received only a craniotomy, the vehicle group underwent $\mathrm{CCl}$ and received an equal volume of vehicle, the JWH group underwent $\mathrm{CCl}$ and received a selective CB2 agonist, JWH133 $(1.5 \mathrm{mg} / \mathrm{kg}$, MedChemExpress, USA), by i.p. injection at $1 \mathrm{~h}$ after operation, and the SR +JWH group underwent $\mathrm{CCl}$ and received SR144528 (3 mg/kg, Cayman, USA) by i.p. injection 3 min before receiving JWH133 intraperitoneally. SR144528 is a selective CB2 antagonist. JWH133 and SR144528 were dissolved in DMSO/ethanol/0.9 \% saline (1:1:18) and injected intraperitoneally into each animal. Untreated animals received an equal volume of vehicle (DMSO/ethanol/0.9\% saline (1:1:18). JWH133 and SR144528 were used according to the methods of previous studies[12, 14].

\section{Immunohistochemistry and quantification}

Immunofluorescence staining was examined $72 \mathrm{~h}$ after surgery, rats $(\mathrm{n}=6$, per group) were euthanized and perfused intracardially with PBS followed by $4 \%$ paraformaldehyde. Brains were removed, postfixed in $4 \%$ paraformaldehyde for $24 \mathrm{~h}$ and dehydrated in a $30 \%$ sucrose solution for $3-5$ days at $4{ }^{\circ} \mathrm{C}$. Coronal brain sections (20- $\mu \mathrm{m}$ thickness) were subjected to immunofluorescence staining. Briefly, after washing with PBS, the sections were permeabilized with $0.4 \%$ Triton X-100 in PBS for 30 min at room temperature, blocked with $10 \%$ goat serum for $1 \mathrm{~h}$ at $37^{\circ} \mathrm{C}$, and subsequently incubated overnight with primary antibodies at $4^{\circ} \mathrm{C}$. After three washes in PBS, the sections were incubated with the appropriate secondary antibodies for $1 \mathrm{~h}$ at $37^{\circ} \mathrm{C}$. The above protocol was repeated once for double staining[14]. The primary antibodies included anti-Iba1 (1:500, Wako, polyclonal囚Japan), anti-CD206 (1:100, Santa Cruz Biotechnologies, monoclonal, USA), anti-CD86 (1:100, Santa Cruz Biotechnologies, Monoclonal, USA), anti-NG2 (1:100, Biorbyt, polyclonal खUK), anti-APC (1:100, Millipore, Monoclonal, USA), anti-myelin basic protein (MBP) (1:100, Millipore, Monoclonal, USA), and anti-neurofilament H (NF-H) (1:100, CST, Monoclonal, USA) antibodies. Confocal microscopy (Zeiss, LSM780, Germany) was used to capture 
images. A minimum of 6 images were captured for each rat from corpus callosum (CC), external capsule (EC) and cortex. The immune-positive cell numbers were calculated with ImageJ software, and presented as the mean number of cells per square millimeter. The immunostaining intensity of MBP, NF200 was used to demonstrate white matter damage after TBI with ImageJ software. All counts were obtained in a blinded fashion[12].

The distribution of microglia around myelin sheaths was assessed by counting the microglial cell bodies in contact with and localized within each myelin sheath in the striatum. The proportion of microglia in contact with myelin relative to the amount of myelin in each image field was calculated. Hence, different amounts of myelin in different fields were considered to represent changes in the redistribution of microglia in relation to myelin[15].

\section{Western blot analysis}

Western blot assays were performed $72 \mathrm{~h}$ after operation. Protein from the pericontusional cortex and corpus callosum area was extracted in RIPA lysis buffer (containing protease and phosphatase inhibitors) prepared as described previously to perform western blotting[11]. Fifty micrograms of prepared protein were loaded into each lane of SDS-PAGE gels. The primary antibodies used in this study included anti-total-PERK (1:1000, CST, Monoclonal, USA), anti-phospho-(p-)PERK (1:1000, Invitrogen, Monoclonal, USA), anti-total-elF2a (1:1000, CST, Monoclonal, USA), anti-p-eIF2a (1:1000, CST, Monoclonal, USA), anti-

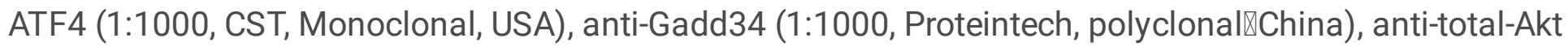
(1:1000, CST, Monoclonal, USA), anti-p-Akt (1:1000, CST, Monoclonal, USA), anti-p-inositol-requiring enzyme 1a (p-IRE1a) (1:1000, Proteintech, polyclonalषChina), anti-activating transcription factor 6 (ATF6) (1:1000, Proteintech, polyclonalषChina), anti-MBP (1:1000, Millipore, Monoclonal, USA), anti-NF-H (1:100, Proteintech, polyclonal $\mathbb{C}$ China), anti-GAPDH (1:1000, Proteintech, Monoclonal, China), and anti- $\beta$-tubulin (1:1000, CST, Monoclonal, USA) antibodies. Briefly, the polyvinylidene difluoride (PVDF) membranes were blocked in $5 \%$ nonfat milk for $1 \mathrm{~h}$ at room temperature and then incubated with primary antibodies at $4^{\circ} \mathrm{C}$ overnight. Subsequently, the membranes were incubated with appropriate secondary antibodies for $1 \mathrm{~h}$ at room temperature. The bands were visualized with a Fusion-FX7 system (Vilber Lourmat, Chongqing, China). ImageJ software was used for densitometric analyses[16].

\section{Real-time polymerase chain reaction}

Total RNA was extracted from pericontusional cortex and EC using RNeasy Mini Kit (Qiagen) according to the manufacturer's instructions ( $72 \mathrm{~h}$ after operation, $\mathrm{n}=6$ per group). One microgram of RNA was reversetranscribed into cDNA using a PrimeScript ${ }^{\mathrm{TM}}$ RT kit (Vazyme, Nanjing, China). RT-PCR systems were set up in $10 \mu$ volumes using a SYBR Premix Ex Taq II kit (Vazyme, Nanjing, China), and RT-PCR was performed on a CFX-96 Real-Time PCR Detection System (Bio-Rad, USA). The program included $30 \mathrm{~s}$ at $95^{\circ} \mathrm{C}, 40$ cycles of $5 \mathrm{~s}$ at $95^{\circ} \mathrm{C}$ and $30 \mathrm{~s}$ at $60^{\circ} \mathrm{C}$, and melt curve analysis. Gene expression was quantified and normalized to that of standard samples (GAPDH)[12]. The sequences of the primers for M1phenotype genes were as follows: CD16 forward sequence, ATCCTAGACCCTGAATGGG; CD16 reverse 
sequence, GCGAGATGAGGCTTTTGT; CD32 forward sequence, CAAAGCCAACCACAGTCA; CD32 reverse sequence, CAATACCGGCAACGAACT; CD86 forward sequence, TGTTGGCCTATCTGCTCTC; and CD86 reverse sequence, AGCTCACTCGGGCTTATG. The sequences of the primers for M2-phenotype genes were as follows: CD163 forward sequence, GGGGTTCCGTCTGTGAT; CD163 reverse sequence, TGTCCGCTTCCTTCTCC; CD206 forward sequence, CAACCAAAGCTGACCAAAG; CD206 reverse sequence, AAGAAATAGGCCGCAACA; Arg1 forward sequence, CAGTATTCACCCCGGCTA; and Arg1 reverse sequence, CCTCTGGTGTCTTCCCAA.

\section{Laser speckle contrast imaging (LSCl)}

LSCl was conducted to image cerebral perfusion and record cerebral blood flow (CBF) $3 \mathrm{~d}$ after TBI ( $\mathrm{n}=6$, per group). The rat body temperature was maintained at $37 \pm 0.5^{\circ} \mathrm{C}$ during the procedure. First, a midline incision was made to expose the skull. Tampons were used to keep the exposure area clean and dry. The head of a PeriCam PSI System was adjusted to ensure that the red cross (indicator laser, $660 \mathrm{~nm}$ ) was located at the center of the brain, and the measurement distance was kept at $10 \mathrm{~cm}$. The test area was adjusted with PIMSoft. Cerebral blood signals were collected at $785 \mathrm{~nm}$ and transferred into blood perfusion images via PIMSoft. Perfusion images were collected with a PeriCam high-resolution LSCI instrument (PSI System, Perimed) with a $70 \mathrm{~mW}$ built-in laser diode for illumination and a CCD camera installed $10 \mathrm{~cm}$ above the skull. Cerebral perfusion was calculated in the region of interest (ROI) located between the bregma and lambda in the hemisphere containing the contusional and pericontusional cortex using PIMSoft software. The CBF change is presented as the mean perfusion value $[17,18]$.

\section{Electrophysiologic recording}

To record the motor evoked potentials (MEPs) elicited by transcranial electrical stimulation 2 weeks after TBI, rats ( $n=6$, per group) were anesthetized with $1 \%$ pentobarbital sodium (25 mg/kg i.p.)[19]. A stimulation needle electrode (DSN1620, Medtronic, USA) was inserted subcutaneously at the base of the nose with the tip touching the scalp, acting as the anode. The cathode was placed at the midpoint of an imaginary line between the two ears. The ground electrode was placed subcutaneously at the base of the tail. The recording electrode was inserted into the contralateral gastrocnemius muscle to record MEPs. Electrical stimulation was performed with a stimulator to excite the brain (Keypoint, Medtronic, USA). A single pulse of stimulation (10 mA, $0.1 \mathrm{~ms}, 1 \mathrm{~Hz}$ ) was delivered by a single electrode. The electrical stimulation was repeated at least five times in each rat with an interval of $15 \mathrm{~s}$. The base-to-peak amplitude of a single stimulation was recorded as the MEP. The MEP and the latency were recorded for analysis[20].

\section{Transmission electron microscopy (TEM)}

TEM was used to measure the thickness of myelin in the corpus callosum (CC)/external capsule (EC) area $3 \mathrm{~d}$ after TBI. Rats ( $\mathrm{n}=6$, per group) were initially perfused with ice-cold saline and subsequently perfused with $4 \%$ PFA and $2.5 \%$ glutaraldehyde in $0.1 \mathrm{~mol} / \mathrm{L} \mathrm{PBS}$ buffer. The CC/EC tissue near the CCI site was microdissected into $1-\mathrm{mm}^{3}$ specimens. Then, these samples were immersion-fixed for $24 \mathrm{~h}$ in $2 \%$ 
glutaraldehyde, washed in PBS and fixed in 1\% osmium tetroxide in $0.1 \mathrm{M}$ PBS for $45 \mathrm{~min}$. The specimens were dehydrated in increasing concentrations of acetone and embedded in Araldite resin. Ultrathin 60-nm sections were cut on a Leica UCT ultramicrotome with a diamond knife (Diatome, Wetzlar, Germany). The prepared ultrathin sections were mounted on copper grids, stained with uranyl acetate and lead citrate, and viewed with a JEM-1400Plus TEM (H-7500, Hitachi Ltd., Japan). Images were acquired in randomly selected CC/EC areas from each section at a magnification of 20,000x. The g-ratios of the myelinated fibers were calculated using ImageJ software by an investigator blinded to the experimental groups, and at least 60 myelinated fibers from each rat were analyzed. The g-ratio was calculated as the ratio of the inner diameter (axon) to the outer diameter (axon and myelin sheath)[21].

\section{DTI scanning and analyses}

DTI was used to evaluate white matter integrity 2 weeks after $\operatorname{TBI}(7,8)$. Rats $(n=6$, per group) were initially anesthetized with $3 \%$ isoflurane and then positioned on an animal cradle with a stereotaxic head holder. Anesthesia was maintained with between $1 \%$ and $1.5 \%$ isoflurane. Respiration and temperature were monitored continuously during the scanning process. A DTI dataset covering the entire brain was collected with the following parameters: TE $=0.85 \mathrm{~ms}, \mathrm{TR}=5000 \mathrm{~ms}, 256 \times 128$ matrix, a total of 32 diffusion sampling directions, $b=826.59 \mathrm{~s} / \mathrm{mm}^{2}$, in-plane resolution $=0.12 \mathrm{~mm}$, and slice thickness $=0.8$ $\mathrm{mm}$. These parameters have been established for acquisition of high-resolution DTI scans in small animals[21].

ParaVision version 5.0 (Bruker BioSpin) was used for analysis of the DTI data. The ROI was drawn manually in a blinded manner to encompass the CC/EC in the ipsilesional hemisphere in order to determine the fractional anisotropy (FA) and radial diffusivity (RD) values. The RD value was calculated using the equation: see equations in supplementary files section.

\section{Behavioral tests}

Behavioral tests were conducted by individuals blinded to the experimental groups $(n=6) 2$ weeks after TBI.

Morris water maze test. Cognitive function was analyzed with the Morris water maze as described previously[21]. Briefly, the rats were trained to search for a hidden platform by using a series of prominent extramaze cues. Nontoxic ink was used to make the water opaque, and the water temperature was maintained at $22-26^{\circ} \mathrm{C}$. In the learning test, the rats were tested for 5 consecutive days, and the circular platform (diameter $=12 \mathrm{~cm}$, height $=20-35 \mathrm{~cm}$ ) was submerged $2 \mathrm{~cm}$ beneath the water surface in a circular pool (diameter $=150 \mathrm{~cm}$, height $=60 \mathrm{~cm}$ ). Each rat was placed into the pool at one of three locations and allowed to locate the hidden platform for $60 \mathrm{~s}$. Each animal was trained in 3 trials (with randomly assigned starting positions) per day to locate the platform. At the end of each trial, the rat was placed on the platform or allowed to stay on the platform for $15 \mathrm{~s}$ with prominent spatial cues displayed around the room. The time spent reaching the platform (learning phase) was recorded. A memory test was performed on day 14 . The platform was removed, and a single $60 \mathrm{~s}$ probe trial was conducted. The 
time spent in the target quadrant where the platform was previously located was recorded. The swim speed of each rat was also recorded to assess gross motor skills during the experiments. The motion trial and behavioral data from the spatial learning test and memory test were recorded and analyzed using a video-based tracking system (ANY-maze, Stoelting, USA).

Open field test. Anxiety-like behavior was evaluated two weeks after TBI in an open field apparatus (100 $\mathrm{cm} \times 100 \mathrm{~cm} \times 40 \mathrm{~cm}$ box). Briefly, a rat was placed in the center of the open field box, and activity was measured and recorded for 5 consecutive minutes using a video-based tracking system (ANY-maze, Stoelting, USA). The total movement time and the time spent in the center of the open field box were analyzed by an investigator who was blinded to the experimental groups[23].

\section{Cell culture}

Primary microglial culture. Primary microglial cultures were prepared as previously described with slight modifications[24]. Briefly, pups were sacrificed, and their brains were isolated. Then, the cerebellum was dissected away from each brain, and the meninges and choroid plexus tissue were separated from the cortical and ventricular surfaces. The cortices were dissected away from the diencephalon, cut into 2-3$\mathrm{mm}^{2}$ pieces and digested with papain and DNase (Sigma) in a $37^{\circ} \mathrm{C}$ water bath for $10 \mathrm{~min}$. After centrifugation (1000 rpm for $5 \mathrm{~min}$ ) and resuspension, the cells were plated in poly-L-lysine (10\%, Solarbio, China)-precoated cell culture flasks and cultured in high-glucose DMEM (Gibco) containing 10\% fetal bovine serum (FBS, Gibco) and 1\% penicillin-streptomycin (Solarbio). After culturing for $10 \mathrm{~d}$ in a humidified atmosphere at $37^{\circ} \mathrm{C}$ containing $5 \% \mathrm{CO}_{2}$ and $95 \%$ air, the cells formed a confluent monolayer (medium changes were performed every $3 \mathrm{~d}$ ). The microglia were separated from the astrocytes by shaking the flask for $4 \mathrm{~h}$ at $220 \mathrm{rpm}$ to detach the layer of loosely adherent astrocytes. The purity of the microglia was $>98 \%$, as verified using anti-lba-1 immunofluorescence staining. Subsequently, the microglia were seeded on poly-L-lysine-precoated coverslips for immunofluorescence analysis.

Primary OL culture. Primary OLs were collected from 1-d-old postnatal rats as described previously with slight modifications[25]. Briefly, pups were sacrificed, and their brains were isolated. Then, the cerebellum was dissected away from each brain, and the meninges and choroid plexus tissue were separated from the cortical and ventricular surfaces. The cortices were dissected away from the diencephalon and cut into $2-3-\mathrm{mm}^{2}$ pieces. Papain and DNase (Sigma) were used for tissue digestion in a $37^{\circ} \mathrm{C}$ water bath for 10 min with occasional inversion. More DNase was added if the tissue became gel-like. The cells were centrifuged at $1000 \mathrm{rpm}$ for $5 \mathrm{~min}$ and resuspended in growth medium (90\% DMEM/F12 (Gibco), 10\% FBS (Gibco), and 1\% penicillin-streptomycin (Solarbio)). Dissociated cells were plated in T-75 culture flasks precoated with poly-L-lysine ( $10 \%$, Solarbio, China). Medium changes were performed every $3 \mathrm{~d}$. On day 6 , the medium was replaced with OL differentiation medium (described below).

To prepare OL differentiation medium, B104 cells were first cultured in medium containing $90 \%$ DMEM/F12 (Gibco), 10\% FBS (Gibco), and 1\% penicillin-streptomycin (Solarbio) until they covered 70$80 \%$ of the flask. The medium was then replaced with N2 medium (98\% DMEM/F12 (Gibco), 1\% penicillin- 
streptomycin (Solarbio), 1\% N2 (Gibco)). Over 3-4 d, the N2 medium slowly became yellowish, indicating that the supernatant contained cytokines. After centrifugation (1000 rpm for $30 \mathrm{~min}$ ) and filtering (0.22 $\mu \mathrm{m}$, Millipore, USA), differentiation medium was prepared with the supernatant (90\% DMEM/F12 (Gibco), 10\% B104 supernatant, and 1\% penicillin-streptomycin (Solarbio)). Differentiation medium changes were performed every $3 \mathrm{~d}$. On day 9 , OLs were obtained for purification.

A transwell system was used to allow communication via diffusible factors and simple conditioned medium (CM) transfer from microglial cultures to OL cultures (Fig 7l). OLs were subjected to oxygenglucose deprivation (OGD) for $2 \mathrm{~h}$ and subsequently returned to normal medium for $24 \mathrm{~h}$ (some OLs were treated with JWH133 (OGD+JWH group) or with vehicle (OGD+ vehicle group) directly). Primary microglia were incubated with vehicle (sham group), tunicamycin $(3 \mu \mathrm{g} / \mathrm{ml})+$ vehicle $(\mathrm{TM}+$ vehicle group), tunicamycin $(3 \mu \mathrm{g} / \mathrm{ml})+\mathrm{JWH} 133(4 \mu \mathrm{M})(\mathrm{TM}+\mathrm{JWH}$ group $)$ or tunicamycin $(3 \mu \mathrm{g} / \mathrm{ml})+\mathrm{SR} 144528(1 \mu \mathrm{M})$ + JWH133 $(4 \mu \mathrm{M})(\mathrm{TM}+\mathrm{SR}+\mathrm{JWH}$ group) for $6 \mathrm{~h}$ and then cocultured with OLs in a transwell system for 24 $\mathrm{h}[14,26,27]$. Subsequent experiments (flow cytometry for apoptosis and immunocytochemical staining for MBP, Iba1 and p-PERK) were performed $24 \mathrm{~h}$ later.

\section{Measurement of volumetric tissue loss and MBP loss}

Rats $(n=6)$ were euthanized 2 weeks after TBI and perfused with PBS followed by $4 \%$ PFA. Subsequently, samples were removed and postfixed in $4 \%$ PFA overnight, transferred to a $25 \%$ sucrose solution until the tissue was submerged at $4^{\circ} \mathrm{C}$, and transferred to a $30 \%$ sucrose solution until the tissue was submerged under the same conditions. Once gradient dehydration was completed, the samples were cut into $25-\mu \mathrm{m}-$ thick sections with a cryostat and stored at $-20^{\circ} \mathrm{C}$. Six equally spaced coronal brain sections encompassing the injury site were selected and stained for MBP (1:100, Millipore, USA) with the same method described previously. Loss of tissue and MBP was analyzed by a blinded observer using NIH Image J software, and the \% of volumetric tissue and MBP loss was calculated as the ratio of the cortical lesion volume to the entire contralateral cortex volume [21, 23].

\section{Statistical analysis}

GraphPad Prism software (version 8.1.0, CA, USA) was used for statistical analyses. The data are presented as the mean \pm SD. Differences between two groups were analyzed by Student's $t$ test (twotailed). The differences in means among multiple groups were analyzed using one-way ANOVA.

Differences in means across groups with repeated measurements over time were analyzed using the repeated-measures ANOVA. When the ANOVA showed significant differences, pairwise comparisons between means were tested by post hoc Bonferroni tests. In all analyses, $P \leq 0.05$ was considered to indicate statistical significance.

\section{Results}

JWH133 shifts microglia/macrophage polarization toward the M2 phenotype after TBI 
To investigate the influence of $\mathrm{CB} 2$ agonism on microglia/macrophage activation and polarization during the acute phase following TBI, we conducted double staining for the microglia marker Iba1 and M1associated or M2-associated markers in the cortex and EC at $3 \mathrm{~d}$ after TBI. As expected, labeling of cells with the M1 marker CD86 was increased after TBI but was significantly decreased by JWH133 (Fig 1A,1B and1E); however, labeling with the M2 marker CD206 was further increased by JWH133 (Fig 1C,1D and $1 F)$. Consistent with the double staining results, RT-PCR of CC/EC samples demonstrated that JWH133 significantly decreased the expression of M1-type genes (CD16, CD32, and CD86) $(P<0.05$, Fig 1G) and increased the expression of M2-type genes (CD163, CD206 and Arg-1) $(P<0.05$, Fig 1H). However, these JWH133-mediated effects were prevented by treatment with SR144528, a CB2 inhibitor.

\section{JWH133 modulates microglia/macrophage polarization through the PERK pathway}

PERK is one of three ER stress sensors on the ER membrane along with IRE1 $\mathrm{a}$ and ATF6, and PERK is autophosphorylated and activated when misfolded proteins accumulate[28]. Previous publications have shown that inhibiting PERK phosphorylation decreases neuronal production of IFN and drives microglia/macrophage polarization toward the M2 phenotype[23]. Therefore, we tested the hypothesis that JWH133 modulates microglia/macrophage polarization through PERK signaling. We first investigated the alterations in PERK and its downstream pathways using western blotting. According to our results, JWH133 downregulated phosphorylation of PERK and its downstream signaling proteins, including elF2a, ATF4 and GADD34, as shown in Fig 2A, 2D, 2E, 2F, and 2G, while it upregulated Akt phosphorylation (Fig 2A and 2H). Notably, phosphorylation of Akt can modulate microglia/macrophage polarization[29]. We also evaluated the influence of JWH133 on ATF6 and p-IRE1a. We found that the expression levels of ATF6 and p-IRE1a were not altered by treatment with JWH133 (Fig 2A, 2B and 2C). These results indicate that this CB2 agonist does not affect ATF6 or IRE1a signaling under our experimental conditions. Taken together, these results support the notion that PERK-mediated activation of Akt polarizes microglia activation toward the M2 phenotype following CB2 activation.

\section{Treatment with JWH133 decreases WMI and demyelination, and reverses tissue loss after TBI}

It has been confirmed that the number of M1 microglial cells increases following TBI in accordance with the severity of WMI, and the M2 phenotype may play a role in neuroprotection[30]. Hence, we hypothesized that JWH133-mediated priming of microglia toward the M2 phenotype after TBI may mitigate WMI. To test our hypothesis, we evaluated the integrity of myelin and axons using immunostaining against MBP and NF200 in the cortex, CC, EC and striatum at $3 \mathrm{~d}$ after TBI with a CB2 agonist and with or without an antagonist. In the sham group, the MBP and NF200 signals in the cortex, $C C, E C$ and striatum were strong $(P<0.05$, Fig 3A-F). However, in the TBI group, both the MBP and NF200 signals were extremely weak in these regions, indicating hypomyelination and axon loss $(P<0.05$, Fig $3 A$ F). Interestingly, the MBP and NF200 levels in the JWH group remained near those of the sham group and were significantly different than those in the TBI group in these areas $(P<0.05$, Fig $3 A-F)$, suggesting that JWH133 promoted maturation of myelin components. To better assess myelin sheath and axon integrity, MBP and NF200 levels in the CC region were measured by lower power image and western blot analysis 
at $3 \mathrm{~d}$ post injury. Consistent with the previous data, TBI induced MBP and NF200 loss, while JWH133 treatment completely abolished these effects $(P<0.05$, Fig 4A-F). These collective findings strongly support the hypothesis that treatment with JWH133 promotes axon and myelin integrity post TBI.

Considering that loss of OLs contributes to impairment of myelin integrity, we investigated whether OLs and OL precursor cells (OPCs) are influenced by JWH133 treatment post TBI. Our data for APC (marker of OLs) and NG2 (markers of OPCs) showed that there were more stained cells in the cortex, CC, and EC regions after JWH133 treatment post TBI than after TBI alone $(P<0.05$, Fig 5A-D).

To further determine whether JWH133 can improve axonal myelination after TBI, myelin thickness was measured in the EC by TEM. The g-ratio (the ratio of the inner axonal diameter to the total outer diameter of the myelin sheath) differed among groups, revealing significant differences in myelin thickness (sham, $0.63 \pm 0.67$; vehicle, $0.78 \pm 0.073 ; \mathrm{JWH}, 0.65 \pm 0.056 ; \mathrm{JWH}+\mathrm{SR}, 0.78 \pm 0.064)$. A significantly increased gratio was observed in the $T B \mathrm{BI}$ group $(\mathrm{P}<0.05$, Fig $5 \mathrm{E}$ and $5 \mathrm{~F})$, suggesting reduced myelin thickness, while JWH133 treatment significantly reduced the g-ratio $(P<0.05$, Fig $5 E$ and 5F). Our data indicate that JWH133 may maintain or promote axonal myelination after TBI.

DTI analysis, which primarily uses water diffusion to yield measures of tissue integrity, was used to identify microstructural damage in an experimental TBI animal model[31]. White matter bundles are composed of collinearly organized and densely packed axons. Net diffusion along the axis of fiber orientation indicates that the tissue is anisotropic; however, WMI may increase diffusion in all directions, making the tissue more isotropic. By assessing diffusion, DTI provides information about the health and architecture of brain white matter tracts. One of the measured variables, FA, represents the degree to which diffusion is restricted to a single direction; higher FA values suggest greater integrity of white matter tracts. On the other hand, RD is a parameter of diffusivity perpendicular to the axon fiber direction, and higher RD values indicate greater abnormality in myelination along the axonal tracts[32]. Similar to previous findings, our results showed that the TBI group (14 d after $\mathrm{CCI}$ ) exhibited a significantly lower FA value and higher RD value in the $E C$ in the hemisphere ipsilateral to the contusion $(P<0.05$, Fig $6 A-C)$ than the sham group. However, treatment with JWH133 significantly restored FA and decreased RD after TBI $(P<0.05$, Fig 6A-C).

To further investigate the biological basis of these DTI data, WMI was quantified by MBP immunostaining. Consistent with the DTI results, there were similar trends between the FA maps and MBP images, and the results showed that JWH133 reduced brain tissue loss and white matter damage after TBI $(P<0.05$, Fig 6D and 6E).

However, all of the described JWH133-mediated effects were prevented by treatment with SR144528.

\section{JWH133 regulates microglial redistribution in the striatum in TBI rats}

Because microglia cell bodies localized around the myelin sheath in the striatum, hence we selected microglia redistribution assessment analysis in the striatum instead of cortex adjacent to area of trauma. 
To investigate the interaction between microglia and OLs after TBI, we firstly confirmed that there was no difference in the total number of microglia between groups ( $P>0.05$, Fig 7A and 7B), interestingly $₫$ the proportion of microglia in contact with myelin fibers (distinguished by MBP staining) was higher in the TBI group ( $3 \mathrm{~d}$ after $\mathrm{CC}$ ) than in the sham group ( $\mathrm{P}<0.05$, Fig $7 \mathrm{~A}$ and $6 \mathrm{C}$ ), indicating that microglia moved toward myelin after TBI. Treatment with JWH133 decreased the proportion of microglia in contact with myelin fibers $(P<0.05$, Fig 7A and 7C). However, SR144528 abolished this effect $(P<0.05$, Fig 7A and 7C). Chemotaxis and phagocytosis are two major behaviors of microglia[15]. We speculated that aberrantly activated microglia redistributed and phagocytosed myelin to aggravate WMI. The results also showed that activation of CB2 protected OLs indirectly through microglia.

\section{JWH133 protects oligodendrocytes indirectly through microglia via the PERK pathway in vitro}

To further investigate the interaction between microglia and OLs, we tested whether JWH133 specifically protects myelin-producing OLs in vitro. We showed that JWH133 provided little direct protection of primary OLs against OGD ( $P>0.05$, Fig $8 \mathrm{~A}$ and $8 \mathrm{~B})$. This finding suggests that the in vivo protection of white matter by JWH133 may be indirect, perhaps mediated by other cell types in the brain; for example, activation of CB2 is known to mitigate the proinflammatory actions of microglia[12]. Thus, we

hypothesized that JWH133 protects OLs via modulation of microglial activation. Consistent with this hypothesis, JWH133 provided profound protection in a transwell system of cocultured microglia and OLs (Fig 8I). We confirmed that the expression of MBP was lower in the group exposed to medium from cultured primary microglia stimulated with tunicamycin than in the sham group $(P<0.05$, Fig $8 C$ and $8 D)$, suggesting that the $\mathrm{CM}$ damaged the cultured OLs. However, the MBP immunostaining intensity was higher in the JWH133-treated group than in the sham group ( $\mathrm{P}<0.05, \mathrm{Fig} 8 \mathrm{C}$ and $8 \mathrm{D})$. Consistent with the MBP immunostaining results, JWH133 decreased $\mathrm{OL}$ apoptosis $(\mathrm{P}<0.05$, Fig $8 \mathrm{G}$ and $8 \mathrm{H})$, as evaluated by flow cytometry. These results indicate that activated microglia may induce WMI via damage to OLs, which also suggests that JWH133 shifts microglial polarization and attenuates WMI.

Next, we further investigated the mechanism by which JWH133 regulates the phenotypic polarization of microglia in vitro. Consistent with the in vivo results, compared with vehicle treatment, treatment with JWH133 abolished the tunicamycin-induced increases in p-PERK levels in microglia $(P<0.05$, Fig $8 E$ and $8 F)$; however, SR144528 abrogated this effect. These results support the notion that activation of CB2 indirectly reduces $\mathrm{OL}$ injury through microglia via the PERK pathway.

\section{Treatment with JWH133 improves cerebral blood flow after TBI}

In the acute phase of TBI, cerebral blood flow decreases significantly[17]. Furthermore, white matter fibers are some of the most vulnerable tissues in TBI, and dramatic changes in cerebral circulation could damage the white matter and affect neurological functions[33]. Prior findings have confirmed that administration of the selective CB2 agonist GP1a enhances cerebral blood flow and improves neurobehavioral outcomes after TBI[18]. Hence, we evaluated cerebral perfusion via LCSI, which can be used as a semiquantitative method. Consistent with our previous findings, perfusion in the lesion areas was obviously lower in the TBI group (at $3 \mathrm{~d}$ following $T B I)$ than in the sham group $(P<0.05$, Figure $9 \mathrm{~A}$ 
and 9B). In addition, cerebral blood flow recovery was promoted in the JWH group compared with the TBI group $(P<0.05$, Figure 9A and 9B). However, the selective CB2 antagonist SR144528 reversed this treatment effect.

\section{Treatment with JWH133 prevents neurological impairments and alleviatesanxiety and depressive-like disorder after TBI}

To assess the recovery of motor function, MEPs were assessed at 14 days post injury, and the amplitudes and MEP latencies were recorded[20]. Compared with the sham group, the TBI group had a lower amplitude $(\mathrm{P}<0.05,10 \mathrm{H}$ and 10I) on the left hindlimb channel. The JWH group exhibited higher amplitudes $(P<0.05,9 \mathrm{H}$ and $9 \mathrm{I})$ than the TBI group, but the SR+JWH group exhibited a lower amplitude for the left hindlimb $(\mathrm{P}<0.05,9 \mathrm{H}$ and $9 \mathrm{I})$ than the JWH group. However, no obvious differences in MEP latency of the left hindlimb were observed among the groups $(P>0.05,10 \mathrm{H}$ and $10 \mathrm{~J})$.

Spatial cognitive functions were evaluated using the Morris water maze test from day 9 to day 14 post TBI (Fig 10A-D)[34], and the escape latencies, quadrant dwell times and swim speeds among the four groups were statistically analyzed. We found that all of the animals in each group showed gradual decreases in escape latency over time $(P<0.05$, Fig 10B). The TBI group exhibited greater learning and memory deficits than the sham group, as manifested by significantly longer escape latencies (indicating loss of spatial learning) $(\mathrm{P}<0.05$, Fig $9 \mathrm{~A}$ and $10 \mathrm{~B})$ and decreased times in the target quadrant (indicating loss of spatial memory) ( $P<0.05$, Fig $10 \mathrm{~A}$ and $10 \mathrm{C})$. The JWH group had a shorter escape latency $(\mathrm{P}<$ 0.05 , Fig $10 \mathrm{~A}$ and $10 \mathrm{~B})$ and greater time in the target quadrant $(\mathrm{P}<0.05$, Fig $10 \mathrm{~A}$ and $10 \mathrm{C})$ than the TBI alone group, which indicated recovery from the learning and memory deficits, but the SR+JWH group showed greater learning and memory deficits $(P<0.05$, Fig 10A-C) than the JWH group. However, animals from all four groups exhibited similar swim speeds, indicating that the memory tests were not confounded by differences in swimming ability $(P>0.05$, Fig 10D).

TBI increases the risk of anxiety, and depressive disorders may be associated with inflammatory reactions in brain regions[35]. Our results support the notion that activation of CB2 reduces neuroinflammation by driving microglial polarization; as a result, anxiety and depressive-like behavior are alleviated after treatment with JWH133. Considering that rodents have a natural aversion to the brightly lit center of an open field, the percentage of time spent in the center versus the periphery of the field can be used as an indicator of anxiety-related behavior. According to our results, the total movement time during the test period was similar among the groups $(P>0.05$, Fig 10F). However, the rats in the TBI group spent significantly less time in the center than those in the sham group $(P<0.05$, Fig $10 \mathrm{E}$ and $10 \mathrm{G})$. These results indicate that the TBI rats exhibited more anxiety-like behavior than the sham rats. Interestingly, rats subjected to TBI and treated with JWH133 spent a greater percentage of time in the central zone than those subjected to $\mathrm{TBI}$ alone $(\mathrm{P}<0.05$, Fig $10 \mathrm{E}$ and $10 \mathrm{G})$, suggesting that activation of CB2 improved anxiety-like behavior following TBI. However, SR144528 pretreatment abolished these effects $(P<0.05$, Fig 10E and 10G). 


\section{Discussion}

At present, there are no effective therapeutic strategies for cognitive and motor deficits in TBI patients[6, 36]. However, numerous works have proven that CB2 agonists can ameliorate brain injury in experimental models of TBI and other diseases, perhaps by regulating microglia-induced neuroinflammatory responses[37, 38]. Taken together with prior findings, our results support the idea that the selective CB2 agonist JWH133 is a promising therapeutic candidate for primary and secondary injury-induced WMI after TBI.

In humans, white matter makes up approximately $60 \%$ of the total brain volume. It is composed mainly of bundled axons and glial cells, including myelin-producing OLs, astrocytes, and microglia. In the brain, large axons are enveloped in the myelin sheath, which greatly promotes neural transmission along nerve fibers. Thus far, research on TBI has traditionally focused on neuronal damage within gray matter, and most preclinical therapies have emphasized protection of neuronal cell bodies in gray matter but have failed to be translated into successful clinical trials[6]. However, increasing evidence has demonstrated a clear correlation between WMI and long-term sensorimotor and cognitive deficits after TBI[39]. Under MRI, WMI in patients with TBI is obviously visible in the internal capsule, CC, and corticospinal tracts (CSTs)[4]. WMI can destroy sensorimotor and cognitive function and elicit neurological dysfunction. Compared with that of focal tissue lesions, the extent of white matter disruption is a better predictor of clinical outcomes[40]. According to our immunostaining, western blot analysis, DTI and TEM results, JWH133 alleviates WMI after TBI and promotes recovery of neurological function.

Despite the crucial connection of WMI to neurological dysfunction, the pathophysiology underlying WMI after TBI remains relatively unknown[41]. A growing amount of evidence has revealed that persistent inflammation is a significant factor that results in sustained white matter degeneration over many years in experimental animal models and humans with TBI. This evidence clearly confirms the existence of an association between chronic microglial activation and neurodegeneration[36] after brain injury. Consistently, in a mouse model of $\mathrm{CCl}$, inflammatory response changes have been found to be accompanied by WMI and loss of myelin[42]. Cooperative M1- and M2-phenotype microglia work to regulate inflammatory responses, scavenge debris, and promote central nervous system (CNS) repair and remodeling after $\mathrm{TBI}$, hence contributing to wound healing and promoting a return to homeostasis after injury. However, it remains controversial whether targeting microglia has a beneficial or detrimental influence on TBI. Thus, enhanced understanding of the dynamic equilibrium between the M1 and M2 phenotypes is needed to enable amelioration of WMI and promotion of post-trauma recovery in brain trauma victims.

Despite the uncertainties, previously reported findings have confirmed that OLs are especially vulnerable to mechanical trauma $[43,44]$ and that neuroinflammatory responses may aggravate WMI following TBI[30, 36]. The current work enhances understanding of WMI pathophysiology by showing that microglia exert protective or destructive influences on OLs depending on their M2 or M1 polarization. However, in OL cultures, JWH133 treatment provided little direct protection in this study. JWH133 
hindered destructive $\mathrm{M} 1$ responses and promoted protective $\mathrm{M} 2$ polarization in microglia, therefore protecting neighboring OLs indirectly.

The underlying mechanisms by which microglia facilitate this progressive loss of white matter and the correlation of white matter loss with OLs remain poorly understood. Thus, we further explored the mechanism of the interaction between microglia and OLs. Recently, ER stress has attracted much attention for its involvement in many CNS diseases, including amyotrophic lateral sclerosis (ALS), Parkinson's disease and TBI[45]. The ER is a structure in which misfolded and nascent proteins are accurately folded by various chaperones (such as GRP78) before transport by secretory vesicles. ER stress is caused by accumulation of misfolded proteins and changes in intracellular calcium levels. ER stress also activates an adaptive signaling pathway for cell survival known as the UPR[28]. The UPR is essentially initiated by ER transmembrane sensors, including PERK, ATF6 and IRE1a[46]. We demonstrated that TBI leads to increases in the phosphorylation levels of PERK and its downstream signaling pathway and that treatment with JWH133 downregulates the phosphorylation of PERK and its downstream signals (eIF2a, ATF4, and GADD34). To further investigate the influence of JWH133 on the PERK pathway in vitro, we treated primary cultured microglia with the ER stress inducer tunicamycin. After treatment with tunicamycin, we confirmed that PERK was activated in the primary cultured microglia and found that treatment with JWH133 obviously suppressed PERK activation. This result suggests that JWH133 can mitigate brain injury by inhibiting the PERK pathway.

ATF4 is a transcription factor that is upregulated when elF2 $a$ is phosphorylated and that elevates the expression of related genes as a recovery mechanism upon ER stress[28]. GADD34 has been shown to bind eukaryotic serine/threonine protein phosphatase, which directly induces elF-2a dephosphorylation[47, 48] and suppresses the UPR[47]. Recently, a study has shown that accumulation of GADD34 directly promotes TBI-induced cell death in a mouse model of TBI[49]. ER stress caused by TBI elicits a transcriptional increase in GADD34, enabling it to bind TRAF6 in competition with Akt. Akt can be ubiquitinated by TRAF6 and facilitate its translocation to the plasma membrane, where it phosphorylates Akt at the T308 position. In cells lacking TRAF6, the processes of ubiquitination, membrane localization and Akt signaling are inevitably impaired[50]. Therefore, binding of GADD34 and TRAF6 inhibits TRAF6-mediated Akt ubiquitination and subsequently prevents membrane translocation and phosphorylation of Akt[51]. The Akt pathway is the main signaling pathway coupled to the CB2 receptor. Moreover, according to our findings, the CB2 agonist JWH133 upregulates Akt phosphorylation, and recent studies have shown that $\mathrm{p}$-Akt can drive microglia from destructive M1 polarization toward beneficial M2 polarization.

We also confirmed that treatment with JWH133 did not alter the expression levels of ATF6 and p-IRE1a and thus did not affect ATF6 or IRE1a signaling under our experimental conditions.

As mentioned above, the present study illuminated the following mechanism: JWH133 downregulates the phosphorylation of PERK and its downstream signals p-elF2a, ATF4, and GADD34 following p-Akt 
upregulation, which mediates a phenotypic shift in microglia and hence exerts a protective effect on neighboring OLs. Importantly, we clarified the interactions between microglia and OLs after TBI.

Notably, mood and anxiety disorders account for a significant part of the disability resulting from TBI[35]. Clinical observations have indicated that a close relationship exists between neuroinflammatory reactions and depression. Although there is an urgent need to investigate the mechanisms of and treatment strategies for these disorders, our present study may provide insights into the pathophysiology of these conditions after TBI.

\section{Conclusions}

In summary, we have demonstrated that a CB2 agonist facilitates OL survival indirectly through PERK pathway-mediated phenotypic translation in microglia. The anti-inflammatory effects of the CB2 agonist and the protection of OLs also significantly improve the milieu of nearby neurons and indirectly exert neuroprotective effects. Therefore, further efforts using CB2 agonists to regulate the PERK axis are warranted in order to prevent the deficits of TBI and other neurological disorders characterized by white matter destruction, such as stroke and neurodegenerative diseases.

\section{Declarations}

\section{Acknowledgments}

We thank Dr. Beike Chen for his experimental technical guidance of DTI, and we would like to acknowledge the service provided by the Chongqing Key Laboratory of Neurology.

\section{Funding}

This work was supported by grants from the National Natural Science Foundation for Youth of China (Grant number 8170051808) and National Natural Science Foundation of China (Grant number 8207052904).

\section{Availability of data and materials}

Data sharing is not applicable to this article as no datasets were generated or analysed during the current study.

\section{Authors' contributions}

LJ and XS conceived and designed the experiments; LL, QL, BS, XY, JZ, and QP performed the experiments; NW , DD and WT analysed the data; LJ and LL wrote and revised the manuscript. All authors read and approved the final manuscript.

\section{Ethics approval and consent to participate}


All animal experiments were performed in accordance with the Animal Research: Reporting of In Vivo Experiments (ARRIVE) ethical guidelines and were approved by the Animal Care and Use Committee of Chongqing Medical University.

\section{Consent for publication}

Not applicable.

\section{Competing interests}

The authors declare that they have no competing interests.

\section{Publisher's Note}

Springer Nature remains neutral with regard to jurisdictional claims in published maps and institutional affiliations.

\section{Abbreviations}

TBI: Traumatic brain injury; CCl: Controlled cortical impact; WMI: white matter injury; CB1: cannabinoid receptors 1; CB2: cannabinoid receptors 2; MBP: myelin basic protein; NF200: neurofilament heavy chain; DTI: diffusion tensor imaging; FA: fractional anisotropy; RD: radial diffusivity; TEM: transmission electron microscopy; OLs: oligodendrocytes; OPCs: OL precursor cells; ER stress: Endoplasmic reticulum stress; UPR: unfolded protein response; ATF6: Activating transcription factor 6; IRE1 a: Inositol-requiring protein 1a; PERK: Protein kinase R (PKR)-like endoplasmic reticulum kinase; elF2a: Eukaryotic translation initiation factor 2a; ATF4: Activating transcription factor 4; GADD34: Growth arrest and DNA damageinducible protein; Akt: Protein kinase B; LSCl: Laser speckle contrast imaging; MEPs: motor evoked potentials; DMEM: Dulbecco's modified eagle medium; FBS: fetal bovine serum; OGD: oxygen-glucose deprivation; RT-PCR: Reverse transcription polymerase chain reaction; CC: corpus callosum; EC: external capsule.

\section{References}

1. Maas AIR, Menon DK, Adelson PD, Andelic N, Bell MJ, Belli A, Bragge P, Brazinova A, Buki A, Chesnut $\mathrm{RM}$, et al: Traumatic brain injury: integrated approaches to improve prevention, clinical care, and research.Lancet Neuro/ 2017, 16:987-1048.

2. Jiang J-Y, Gao G-Y, Feng J-F, Mao Q, Chen L-G, Yang X-F, Liu J-F, Wang Y-H, Qiu B-H, Huang X-J: Traumatic brain injury in China. The Lancet Neurology 2019, 18:286-295.

3. Spitz G, Maller JJ, O'Sullivan R, Ponsford JL: White matter integrity following traumatic brain injury: the association with severity of injury and cognitive functioning.Brain Topogr 2013, 26:648-660.

4. Kinnunen KM, Greenwood R, Powell JH, Leech R, Hawkins PC, Bonnelle V, Patel MC, Counsell SJ, Sharp DJ: White matter damage and cognitive impairment after traumatic brain injury.Brain 2011, 
134:449-463.

5. Betz J, Zhuo J, Roy A, Shanmuganathan K, Gullapalli RP: Prognostic value of diffusion tensor imaging parameters in severe traumatic brain injury.J Neurotrauma 2012, 29:1292-1305.

6. Loane DJ, Faden Al: Neuroprotection for traumatic brain injury: translational challenges and emerging therapeutic strategies. Trends Pharmacol Sci 2010, 31:596-604.

7. Cristino L, Bisogno T, Di Marzo V: Cannabinoids and the expanded endocannabinoid system in neurological disorders.Nature Reviews Neurology 2019, 16:9-29.

8. Zarruk JG, Fernandez-Lopez D, Garcia-Yebenes I, Garcia-Gutierrez MS, Vivancos J, Nombela F, Torres M, Burguete MC, Manzanares J, Lizasoain I, Moro MA: Cannabinoid type 2 receptor activation downregulates stroke-induced classic and alternative brain macrophage/microglial activation concomitant to neuroprotection.Stroke 2012, 43:211-219.

9. Tan HP, Guo Q, Hua G, Chen JX, Liang JC: Inhibition of endoplasmic reticulum stress alleviates secondary injury after traumatic brain injury.Neural Regen Res 2018, 13:827-836.

10. Sen T, Gupta R, Kaiser H, Sen N: Activation of PERK Elicits Memory Impairment through Inactivation of CREB and Downregulation of PSD95 After Traumatic Brain Injury. The Journal of Neuroscience 2017, 37:5900-5911.

11. Li L, Tao Y, Tang J, Chen Q, Yang Y, Feng Z, Chen Y, Yang L, Yang Y, Zhu G, et al: A Cannabinoid Receptor 2 Agonist Prevents Thrombin-Induced Blood-Brain Barrier Damage via the Inhibition of Microglial Activation and Matrix Metalloproteinase Expression in Rats. Translational Stroke Research 2015, 6:467-477.

12. Lin L, Yihao T, Zhou F, Yin N, Qiang T, Haowen Z, Qianwei C, Jun T, Yuan Z, Gang Z, et al: Inflammatory Regulation by Driving Microglial M2 Polarization: Neuroprotective Effects of Cannabinoid Receptor-2 Activation in Intracerebral Hemorrhage. Front Immunol 2017, 8:112.

13. Wu J, He J, Tian X, Zhong J, Li H, Sun X: Activation of the Hedgehog Pathway Promotes Recovery of Neurological Function After Traumatic Brain Injury by Protecting the Neurovascular Unit.Translational Stroke Research 2020, 11:720-733.

14. Li L, Yun D, Zhang Y, Tao Y, Tan Q, Qiao F, Luo B, Liu Y, Fan R, Xian J, Yu A: A cannabinoid receptor 2 agonist reduces blood-brain barrier damage via induction of MKP-1 after intracerebral hemorrhage in rats.Brain Res 2018, 1697:113-123.

15. Zhang L-Y, Pan J, Mamtilahun M, Zhu Y, Wang L, Venkatesh A, Shi R, Tu X, Jin K, Wang Y, et al: Microglia exacerbate white matter injury via complement C3/C3aR pathway after hypoperfusion. Theranostics 2020, 10:74-90.

16. Huang ZJ, Cao F, Wu Y, Peng JH, Zhong JJ, Jiang Y, Yin C, Guo ZD, Sun XC, Jiang L, Cheng CJ: Apolipoprotein E promotes white matter remodeling via the Dab1-dependent pathway after traumatic brain injury. CNS Neuroscience \& Therapeutics 2020, 26:698-710.

17. Liu H, He J, Zhang Z, Liu L, Huo G, Sun X, Cheng C: Evolution of cerebral perfusion in the pericontusional cortex in mice revealed by in vivo laser speckle imaging after traumatic brain injury. Brain Research 2018, 1700:118-125. 
18. Braun M, Khan ZT, Khan MB, Kumar M, Ward A, Achyut BR, Arbab AS, Hess DC, Hoda MN, Baban B, et al: Selective activation of cannabinoid receptor-2 reduces neuroinflammation after traumatic brain injury via alternative macrophage polarization.Brain Behav Immun 2018, 68:224-237.

19. Xia M, Chen W, Wang J, Yin Y, Guo C, Li C, Li M, Tang X, Jia Z, Hu R, et al: TRPA1 Activation-Induced Myelin Degradation Plays a Key Role in Motor Dysfunction After Intracerebral Hemorrhage.Frontiers in Molecular Neuroscience 2019, 12.

20. Zhang J, Liu X, Ma K, Chen M, Xu H, Niu X, Gu H, Wang R, Chen X, Sun H: Collagen/heparin scaffold combined with vascular endothelial growth factor promotes the repair of neurological function in rats with traumatic brain injury.Biomaterials Science 2021.

21. Zhang Q, Zhu W, Xu F, Dai X, Shi L, Cai W, Mu H, Hitchens TK, Foley LM, Liu X, et al: The interleukin4/PPARgamma signaling axis promotes oligodendrocyte differentiation and remyelination after brain injury.PLoS Biol 2019, 17:e3000330.

22. Chen B, Tan Q, Zhao W, Yang Q, Zhang H, Gao F, Liu X, Feng H, Jiang D: Diffusion tensor imaging and electrophysiology as robust assays to evaluate the severity of acute spinal cord injury in rats. $B M C$ Neurol 2020, 20:236.

23. Sen T, Saha P, Gupta R, Foley LM, Jiang T, Abakumova OS, Hitchens TK, Sen N: Aberrant ER Stress Induced Neuronal-IFN $\beta$ Elicits White Matter Injury Due to Microglial Activation and T-Cell Infiltration after TBI. The Journal of Neuroscience 2020, 40:424-446.

24. Wang Y-w, Zhou Q, Zhang X, Qian Q-q, Xu J-w, Ni P-f, Qian Y-n: Mild endoplasmic reticulum stress ameliorates lipopolysaccharide-induced neuroinflammation and cognitive impairment via regulation of microglial polarization.Journal of Neuroinflammation 2017, 14.

25. Pu A, Mishra MK, Dong Y, Ghorbanigazar S, Stephenson EL, Rawji KS, Silva C, Kitagawa H, Sawcer S, Yong VW: The glycosyltransferase EXTL2 promotes proteoglycan deposition and injurious neuroinflammation following demyelination.Journal of Neuroinflammation 2020, 17.

26. Wang YW, Zhou Q, Zhang X, Qian QQ, Xu JW, Ni PF, Qian YN: Mild endoplasmic reticulum stress ameliorates lipopolysaccharide-induced neuroinflammation and cognitive impairment via regulation of microglial polarization.J Neuroinflammation 2017, 14:233.

27. Tang J, Miao H, Jiang B, Chen Q, Tan L, Tao Y, Zhang J, Gao F, Feng H, Zhu G, Chen Z: A selective CB2R agonist (JWH133) restores neuronal circuit after Germinal Matrix Hemorrhage in the preterm via CX3CR1+ microglia.Neuropharmacology 2017, 119:157-169.

28. Shin YH, Cho H, Choi BY, Kim J, Ha J, Suh SW, Park SB: Phenotypic Discovery of Neuroprotective Agents by Regulation of Tau Proteostasis via Stress-Responsive Activation of PERK Signaling.Angew Chem Int Ed Engl 2021, 60:1831-1838.

29. Wang G, Shi Y, Jiang X, Leak RK, Hu X, Wu Y, Pu H, Li W-W, Tang B, Wang Y, et al: HDAC inhibition prevents white matter injury by modulating microglia/macrophage polarization through the GSK3ß/PTEN/Akt axis.Proceedings of the National Academy of Sciences 2015, 112:2853-2858.

30. Wang G, Zhang J, Hu X, Zhang L, Mao L, Jiang X, Liou AK, Leak RK, Gao Y, Chen J: Microglia/macrophage polarization dynamics in white matter after traumatic brain injury.J Cereb 
Blood Flow Metab 2013, 33:1864-1874.

31. Hoogenboom WS, Rubin TG, Ye K, Cui MH, Branch KC, Liu J, Branch CA, Lipton ML: Diffusion Tensor Imaging of the Evolving Response to Mild Traumatic Brain Injury in Rats.J Exp Neurosci 2019, 13:1179069519858627.

32. Liu X, Kinoshita M, Shinohara H, Hori O, Ozaki N, Hatta T, Honma S, Nakada M: Direct evidence of the relationship between brain metastatic adenocarcinoma and white matter fibers: A fiber dissection and diffusion tensor imaging tractography study.J Clin Neurosci 2020, 77:55-61.

33. Nogge G, Giannetti M: Midgut absorption of undigested albumin and other proteins by tsetse, Glossina M. morsitans (Diptera: Glossinidae).J Med Entomo/ 1979, 16:263.

34. Vekaria HJ, Hubbard WB, Scholpa NE, Spry ML, Gooch JL, Prince SJ, Schnellmann RG, Sullivan PG: Formoterol, a $\beta 2$-adrenoreceptor agonist, induces mitochondrial biogenesis and promotes cognitive recovery after traumatic brain injury.Neurobiology of Disease 2020, 140.

35. Broussard JI, Acion L, De Jesus-Cortes H, Yin T, Britt JK, Salas R, Costa-Mattioli M, Robertson C, Pieper AA, Arciniegas DB, Jorge R: Repeated mild traumatic brain injury produces neuroinflammation, anxiety-like behaviour and impaired spatial memory in mice.Brain Inj 2018, 32:113-122.

36. Johnson VE, Stewart JE, Begbie FD, Trojanowski JQ, Smith DH, Stewart W: Inflammation and white matter degeneration persist for years after a single traumatic brain injury.Brain 2013, 136:28-42.

37. Zhang B, West EJ, Van KC, Gurkoff GG, Zhou J, Zhang XM, Kozikowski AP, Lyeth BG: HDAC inhibitor increases histone $\mathrm{H} 3$ acetylation and reduces microglia inflammatory response following traumatic brain injury in rats. Brain Res 2008, 1226:181-191.

38. Dash PK, Orsi SA, Moore AN: Histone deactylase inhibition combined with behavioral therapy enhances learning and memory following traumatic brain injury.Neuroscience 2009, 163:1-8.

39. Xia Y, Pu H, Leak RK, Shi Y, Mu H, Hu X, Lu Z, Foley LM, Hitchens TK, Dixon CE, et al: Tissue plasminogen activator promotes white matter integrity and functional recovery in a murine model of traumatic brain injury.Proceedings of the National Academy of Sciences 2018, 115:E9230-E9238.

40. Sidaros A, Engberg AW, Sidaros K, Liptrot MG, Herning M, Petersen P, Paulson OB, Jernigan TL, Rostrup E: Diffusion tensor imaging during recovery from severe traumatic brain injury and relation to clinical outcome: a longitudinal study.Brain 2008, 131:559-572.

41. Hellyer PJ, Leech R, Ham TE, Bonnelle V, Sharp DJ: Individual prediction of white matter injury following traumatic brain injury.Ann Neurol 2013, 73:489-499.

42. Faden Al, Wu J, Stoica BA, Loane DJ: Progressive inflammation-mediated neurodegeneration after traumatic brain or spinal cord injury.Br J Pharmacol 2016, 173:681-691.

43. Wang G, Jiang X, Pu H, Zhang W, An C, Hu X, Liou AK, Leak RK, Gao Y, Chen J: Scriptaid, a novel histone deacetylase inhibitor, protects against traumatic brain injury via modulation of PTEN and AKT pathway : scriptaid protects against TBI via AKT.Neurotherapeutics 2013, 10:124-142.

44. Wang GH, Jiang ZL, Li YC, Li X, Shi H, Gao YQ, Vosler PS, Chen J: Free-radical scavenger edaravone treatment confers neuroprotection against traumatic brain injury in rats.J Neurotrauma 2011, 28:2123-2134. 
45. Liew HK, Hu WF, Lin PB, Wang PK, Tsai AP, Pang CY, Chen TY: Over-Activated Proteasome Mediates Neuroinflammation on Acute Intracerebral Hemorrhage in Rats.Cells 2019, 8.

46. Kim I, Xu W, Reed JC: Cell death and endoplasmic reticulum stress: disease relevance and therapeutic opportunities.Nat Rev Drug Discov 2008, 7:1013-1030.

47. Connor JH, Weiser DC, Li S, Hallenbeck JM, Shenolikar S: Growth arrest and DNA damage-inducible protein GADD34 assembles a novel signaling complex containing protein phosphatase 1 and inhibitor 1.Mol Cell Biol 2001, 21:6841-6850.

48. Novoa I, Zeng H, Harding HP, Ron D: Feedback inhibition of the unfolded protein response by GADD34-mediated dephosphorylation of elF2alpha.J Cell Biol 2001, 153:1011-1022.

49. Sen N: ER Stress, CREB, and Memory: A Tangled Emerging Link in Disease.Neuroscientist 2019, 25:420-433.

50. Yang WL, Wang J, Chan CH, Lee SW, Campos AD, Lamothe B, Hur L, Grabiner BC, Lin X, Darnay BG, Lin HK: The E3 ligase TRAF6 regulates Akt ubiquitination and activation.Science 2009, 325:11341138.

51. Farook JM, Shields J, Tawfik A, Markand S, Sen T, Smith SB, Brann D, Dhandapani KM, Sen N: GADD34 induces cell death through inactivation of Akt following traumatic brain injury.Cell Death Dis 2013, 4:e754.

\section{Figures}



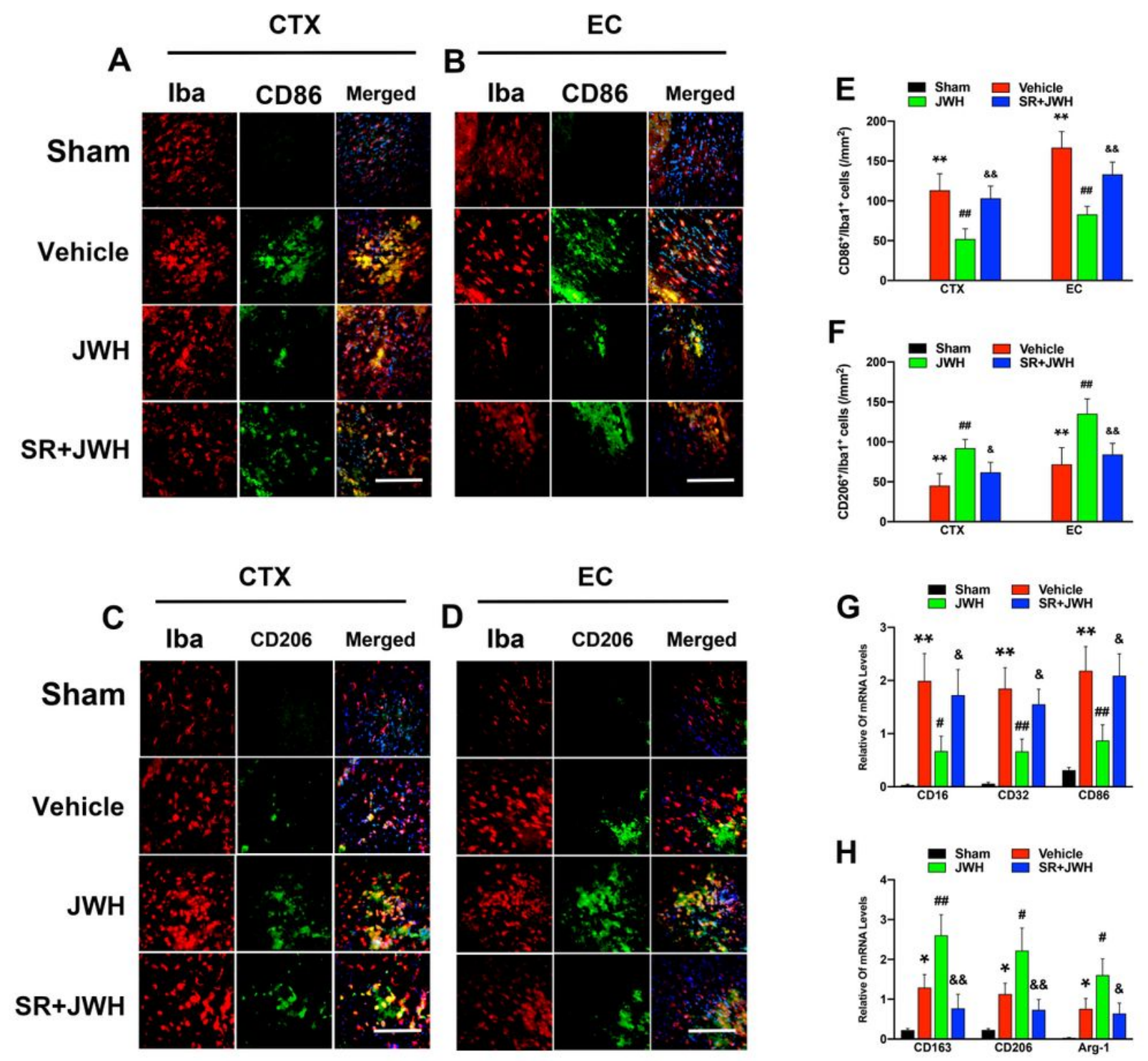

\section{Figure 1}

A CB2 agonist primes microglia toward the M2 phenotype (A-D) Double immunofluorescence staining (scale bars $=40 \mu \mathrm{m}$ ) for the M1 marker CD86 $(A, B)$ or the M2 marker CD206 $(C, D)$ with the microglial marker Iba1 in the ipsilesional cortex and EC at $3 \mathrm{~d}$ following TBI. Iba1/CD86-dual labeled M1 microglia (E) and Iba1/CD206-dual labeled M2 microglia (F) were quantified and expressed as cells/mm2. (G, H) mRNA levels of M1-associated genes, including CD16, CD32, and CD86 (G), and M2-associated genes, including CD163, CD206, and Arg-1 (H). The data are expressed as the means \pm SDs, $n=6 .{ }^{*} P<0.05$ and $* \star \mathrm{P}<0.01$ compared to the sham group, $\# \mathrm{P}<0.05$ and \#\#P $<0.01$ compared to the vehicle group, and \&P $<0.05$ and \&\&P $<0.01$ compared to the JWH group. 


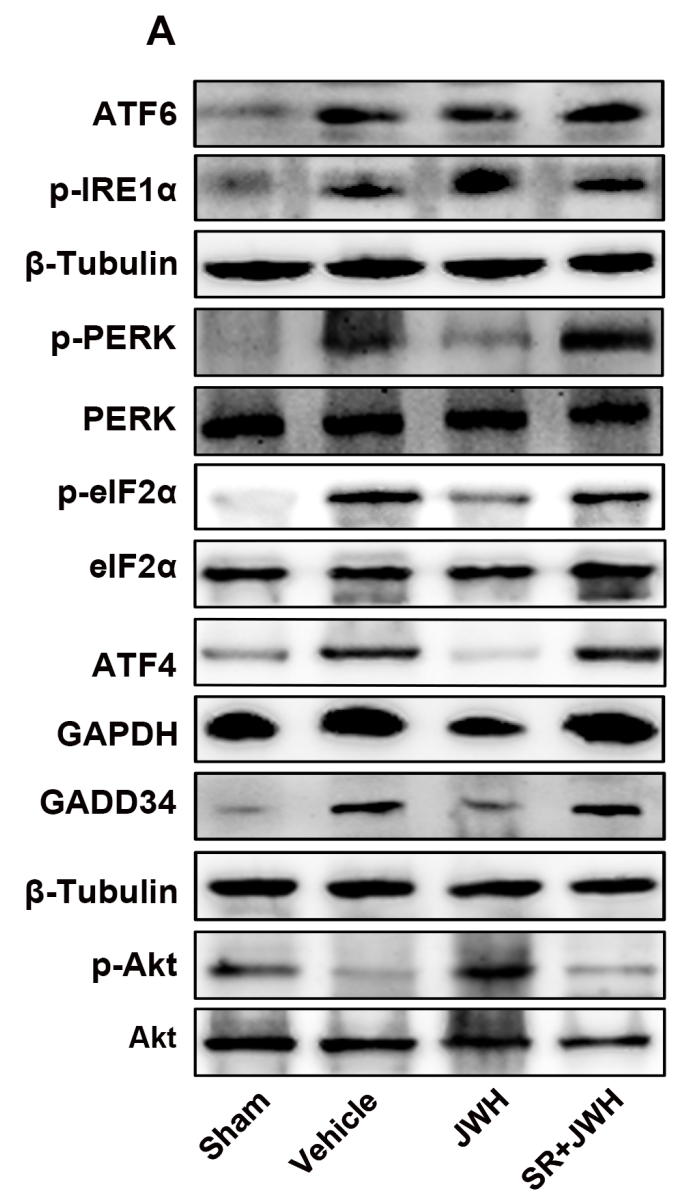

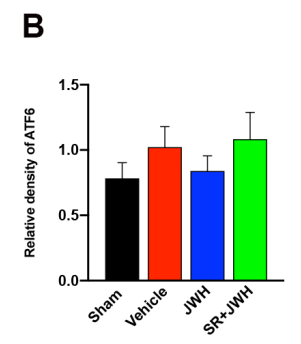

E

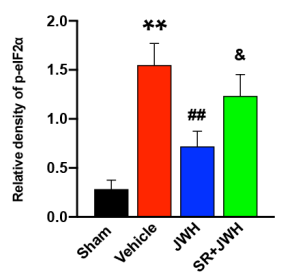

C

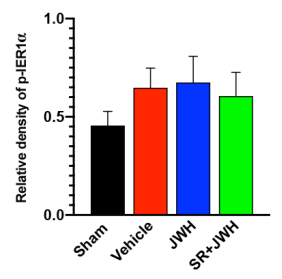

$\mathbf{F}$

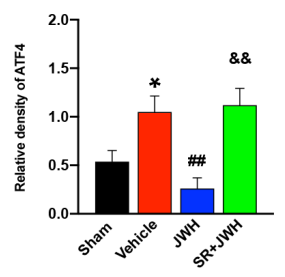

H

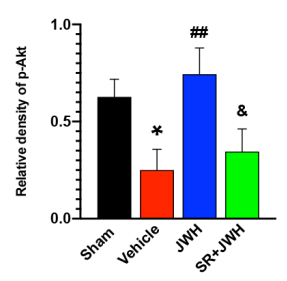

D

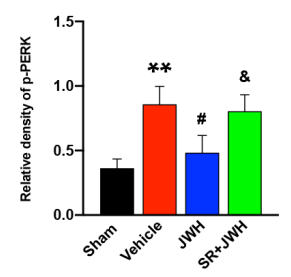

G

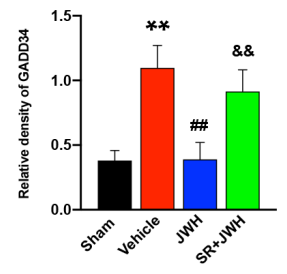

\section{Figure 2}

A CB2 agonist modulates microglia/macrophage polarization through the PERK pathway. (A, D-G) JWH133 inhibited the phosphorylation of PERK and its downstream signals p-elF2a, ATF4 and GADD34 at $3 \mathrm{~d}$ following TBI. $(\mathrm{A}, \mathrm{H}) \mathrm{JWH} 133$ prevented the loss of $\mathrm{p}$-Akt. The data are expressed as the mean \pm SDs, $n=6 .{ }^{*} P<0.05$ and ${ }^{*} \mathrm{P}<0.01$ compared to the sham group, $\# \mathrm{P}<0.05$ and $\# \# \mathrm{P}<0.01$ compared to the vehicle group, and \&P $<0.05$ and \&\&P $<0.01$ compared to the JWH group. (A-C) JWH133 did not alter the expression levels of ATF6 and p-IRE1 $a$. The data are expressed as the means \pm SDs, $n=6$. $P>0.05$. 

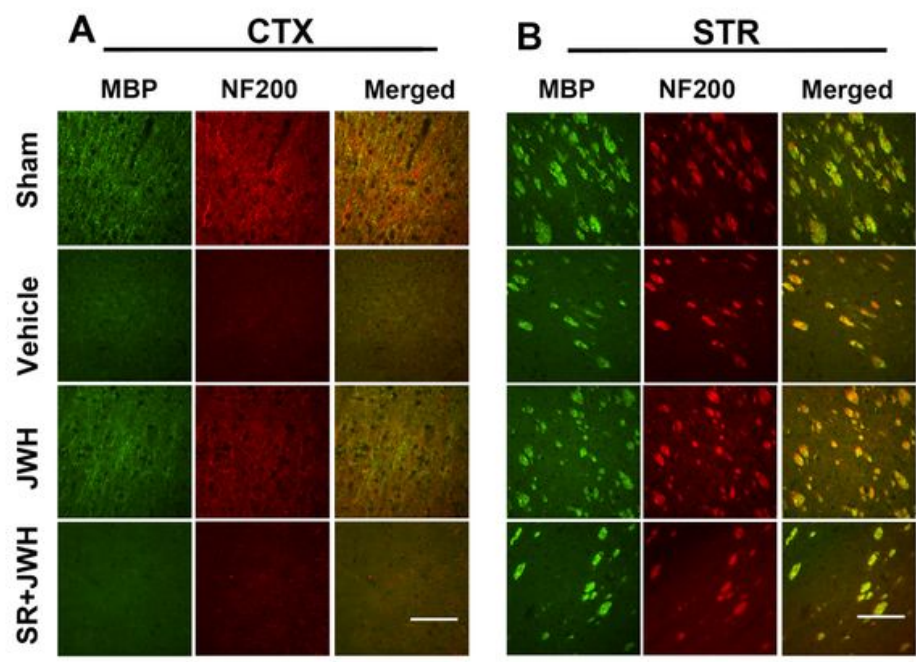

E
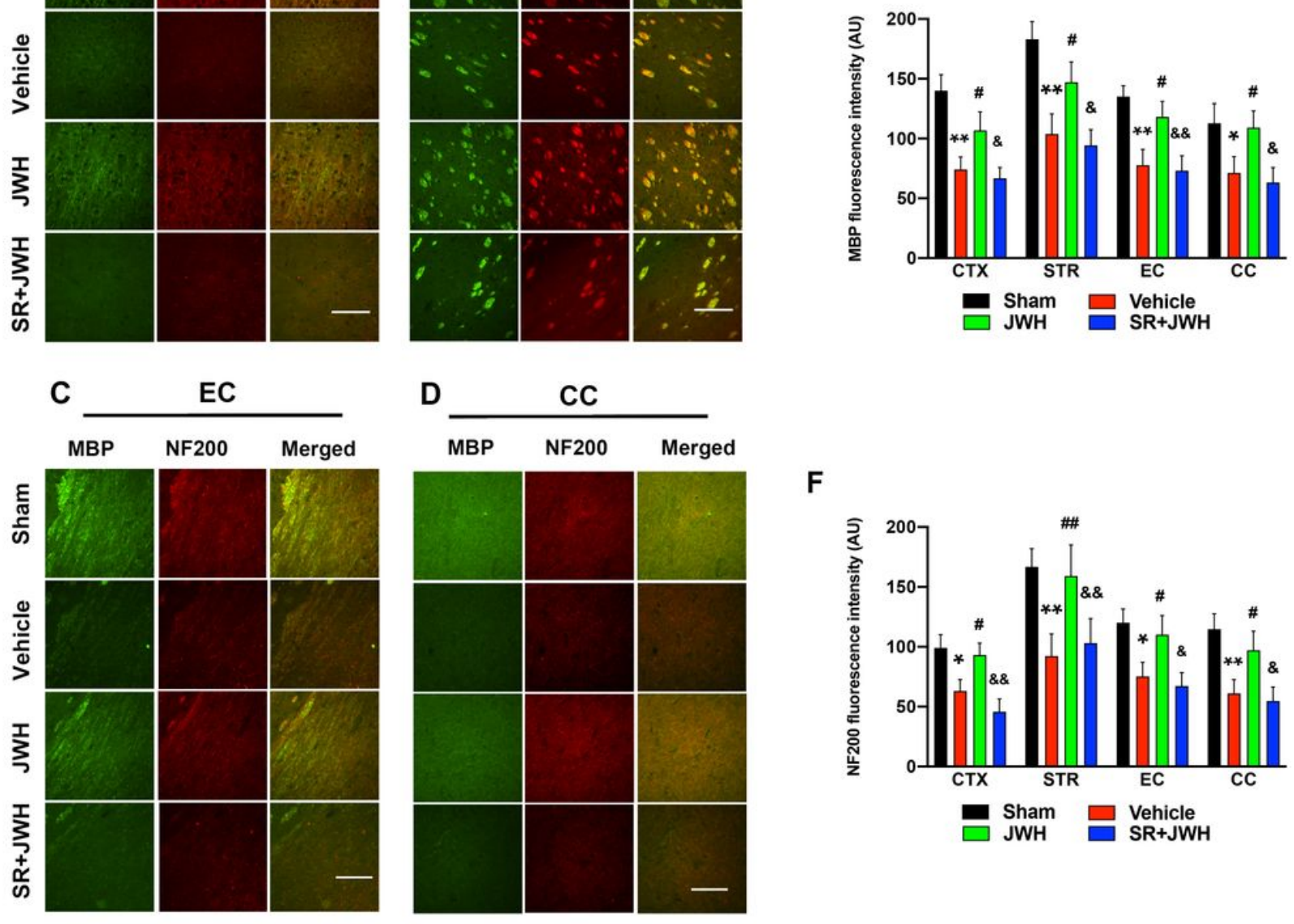

F

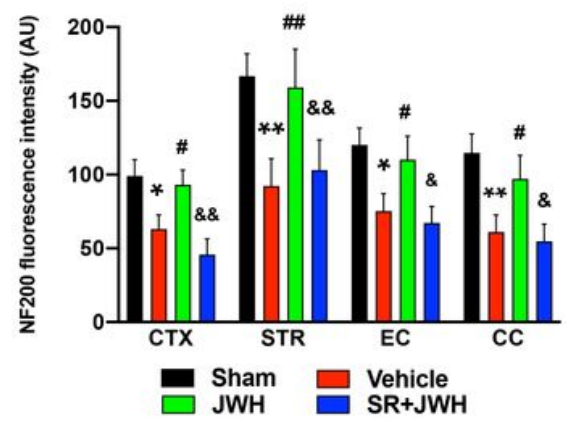

Figure 3

A CB2 agonist protects the integrity of myelin and axons at $3 \mathrm{~d}$ following TBI. (A-F) JWH133 enhanced the expression of MBP and NF200 in the cortex, CC, EC and striatum, as assessed by immunostaining (scale bars $=40 \mu \mathrm{m}$ ). The data are expressed as the means $\pm \mathrm{SDs}, \mathrm{n}=6$. ${ }^{*} \mathrm{P}<0.05$ and $* * \mathrm{P}<0.01$ compared to the sham group, \#P $<0.05$ and \#\#P $<0.01$ compared to the vehicle group, and \&P $<0.05$ and \&\&P $<$ 0.01 compared to the JWH group. 


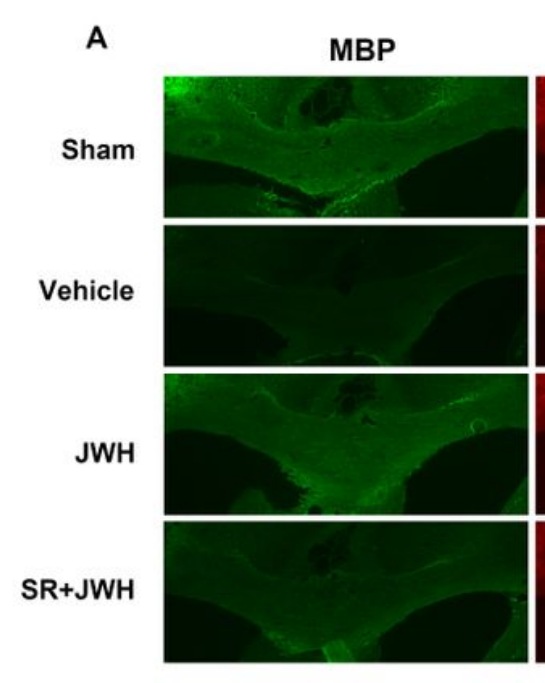

B

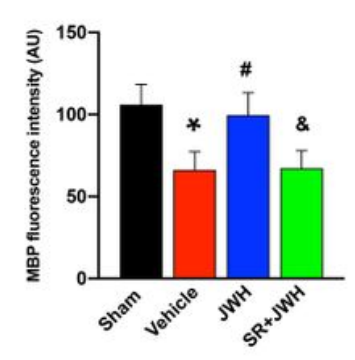

NF200
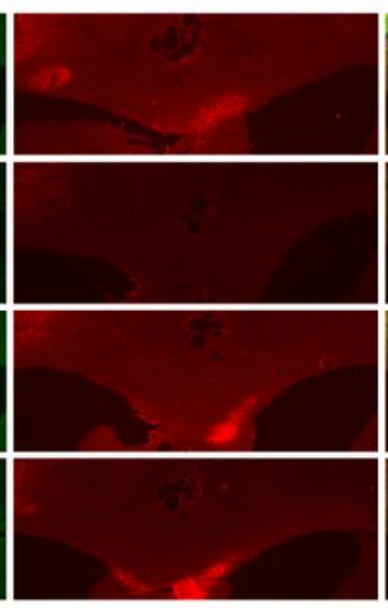

C

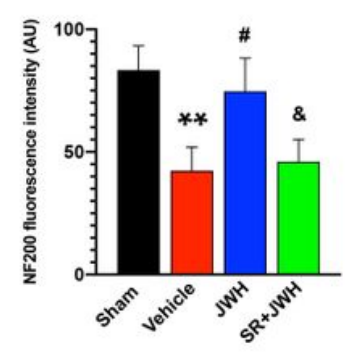

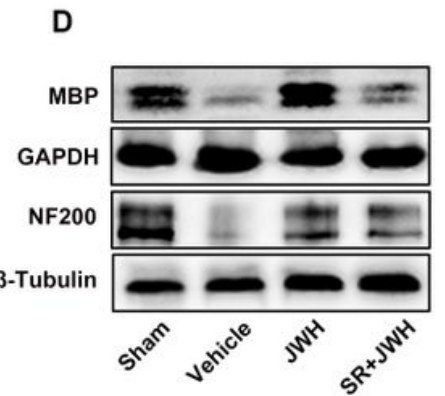

E

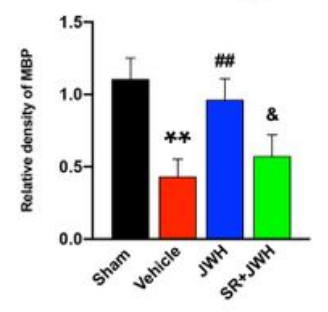

$\mathbf{F}$

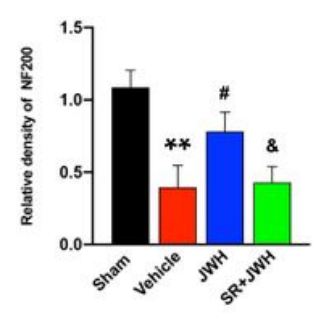

\section{Figure 4}

A CB2 agonist alleviates white matter damage at $3 \mathrm{~d}$ following TBI. (A-C) JWH133 enhanced the expression of MBP and NF200 in the CC area as assessed by lower power image (scale bars $=10 \mu \mathrm{m}$ ). (DF) JWH133 prevented the decreases in MBP and NF200 in the CC area, as indicated by western blot analysis. The data are expressed as the means $\pm S D s, n=6$. ${ }^{*} P<0.05$ and $* * P<0.01$ compared to the sham group, \#P<0.05 and \#\#P<0.01 compared to the vehicle group, and \&P $<0.05$ and \&\&P 0.01 compared to the JWH group. 

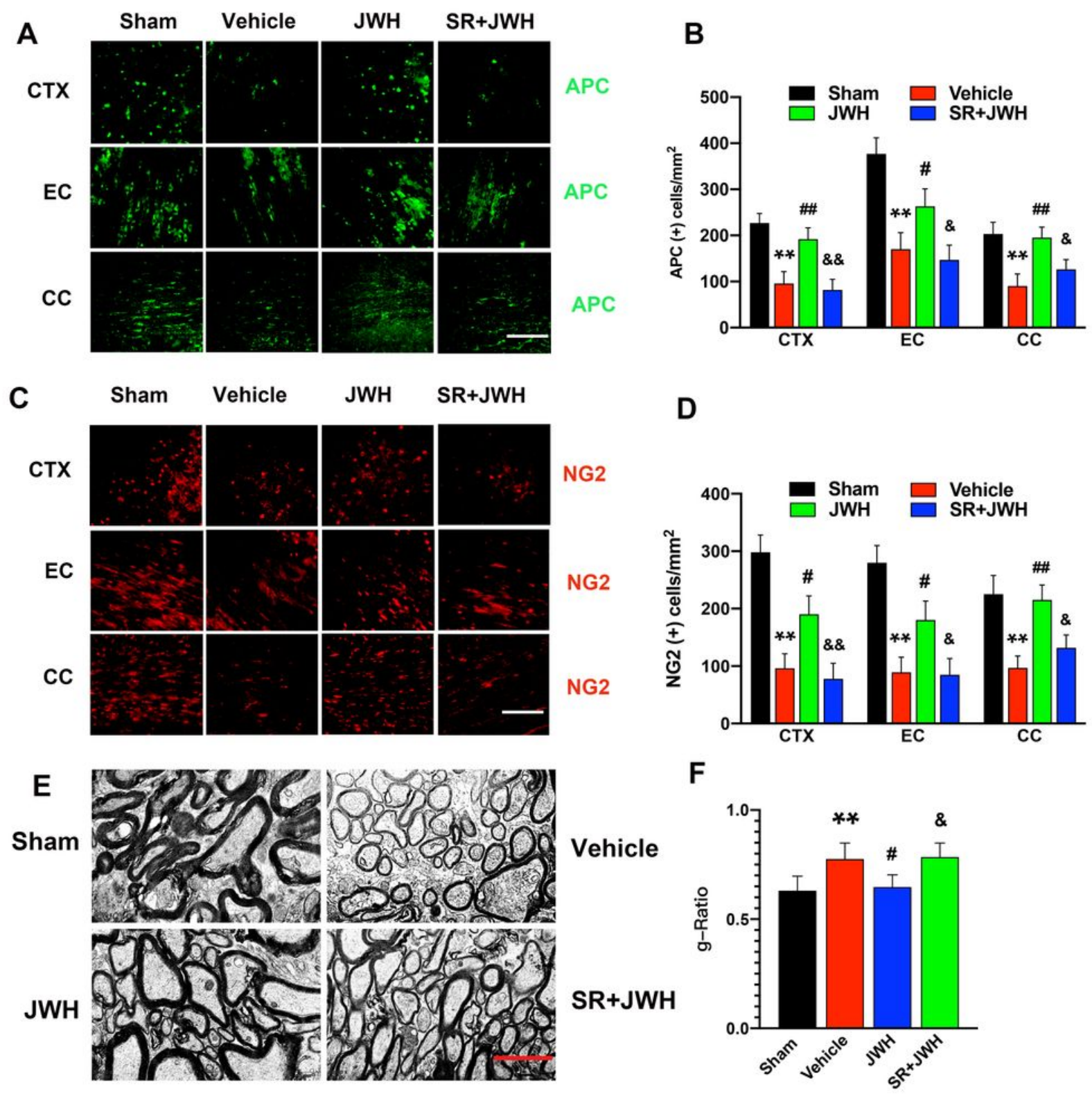

\section{Figure 5}

A CB2 agonist prevents loss of myelin-producing OLs and OPCs. In addition, the CB2 agonist inhibits demyelination at $3 \mathrm{~d}$ following TBI. (A-D) JWH133 increased the number of cells positive for NG2 (a marker of OPCs) and APC (a marker of OLs) in the cortex, CC, and EC (scale bars $=40 \mu \mathrm{m}$ ). The data are expressed as the means $\pm \mathrm{SDs}, \mathrm{n}=6 .{ }^{*} \mathrm{P}<0.05$ and ${ }^{*} \mathrm{P}<0.01$ compared to the sham group, $\# \mathrm{P}<0.05$ and $\# \# P<0.01$ compared to the vehicle group, and \&P $<0.05$ and \&\&P $<0.01$ compared to the JWH group. $(E$, F) JWH133 increased myelin thickness in the CC/EC, as visualized by TEM (scale bars $=1 \mu \mathrm{m}$ ). The data 
are expressed as the means \pm SDs, $n=60$ axons from 6 animals each group. ${ }^{\star *} \mathrm{P}<0.01$ compared to the sham group, \#P $<0.05$ compared to the vehicle group, and \&P $<0.05$ compared to the JWH group.

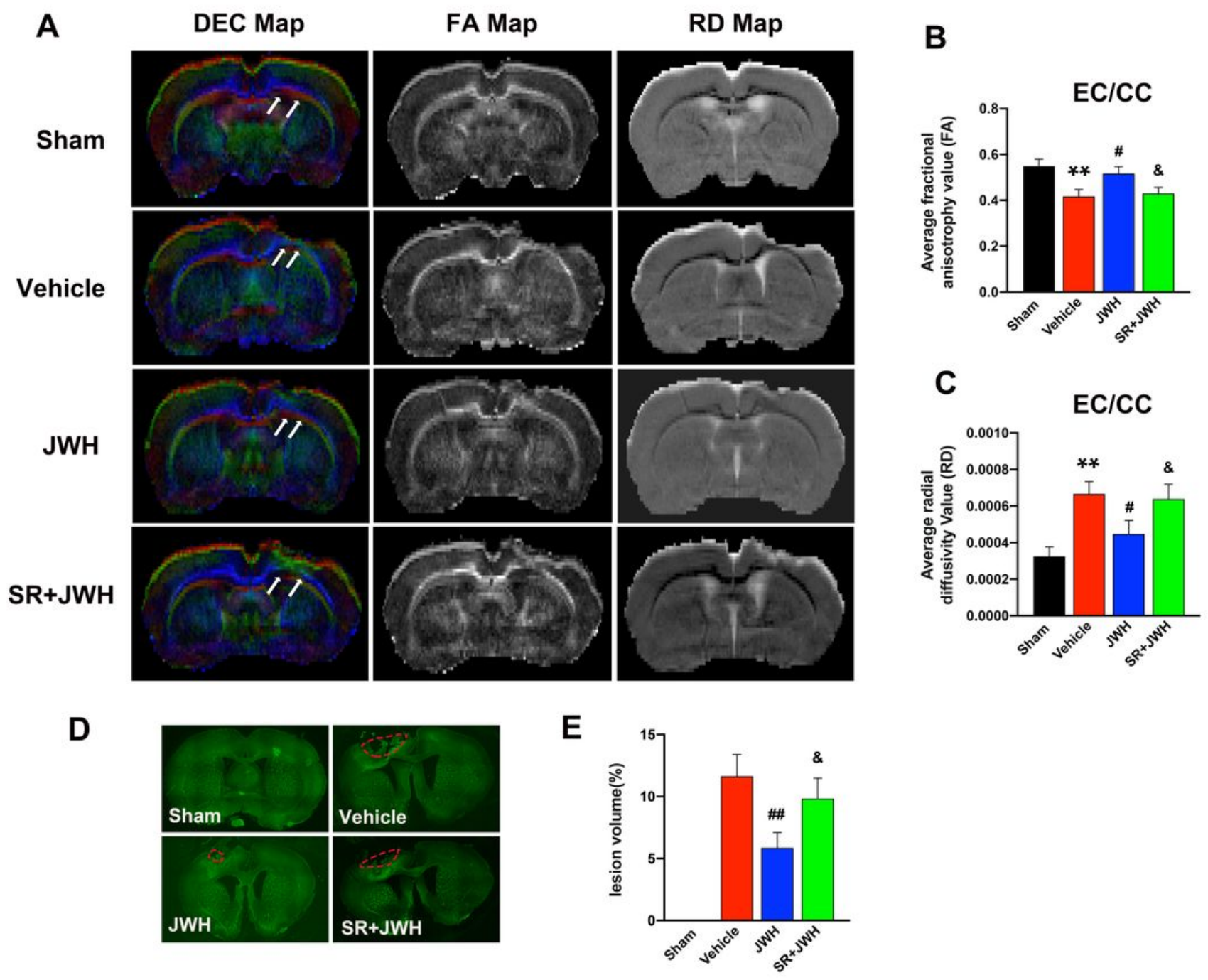

\section{Figure 6}

A CB2 agonist reduces WMI, as measured by DTI and assessment of lesion volumes. (A) JWH133 attenuated $\mathrm{WMI}$, as determined by in vivo MRI, at $14 \mathrm{~d}$ after TBI. Panel A shows representative RD, FA and DEC maps centered on the site of contusion. The Directionally encoded color (DEC) maps indicate the direction of water diffusion (red, mediolateral; green, dorsoventral; blue, anteroposterior). (B) Quantification of average fractional anisotropy (FA) and radial diffusivity (RD) values in the CC/EC areas of the ipsilateral hemispheres (A) JWH133 attenuated WMI, as determined by in vivo MRI, at $14 \mathrm{~d}$ after TBI. Panel A shows representative RD, FA and DEC maps centered on the site of contusion. The Directionally encoded color (DEC) maps indicate the direction of water diffusion (red, mediolateral; green, dorsoventral; blue, anteroposterior). (B) Quantification of average fractional anisotropy (FA) and radial diffusivity (RD) values in the CC/EC areas of the ipsilateral hemispheres (The measurement areas were 
indicated by white arrows). The values indicate that the FA and RD values were rescued after JWH133 treatment. (D, E) JWH133 reduced brain tissue loss and white matter damage at $14 \mathrm{~d}$ after TBI. Scale bar $=10 \mu \mathrm{m}$. The data are expressed as the means \pm SDs, $n=6$. ${ }^{*}<0.05$ and $* * P<0.01$ compared to the sham group, \#P $<0.05$ and \#\#P $<0.01$ compared to the vehicle group, and \&P $<0.05$ and \&\&P 0.01 compared to the JWH group.

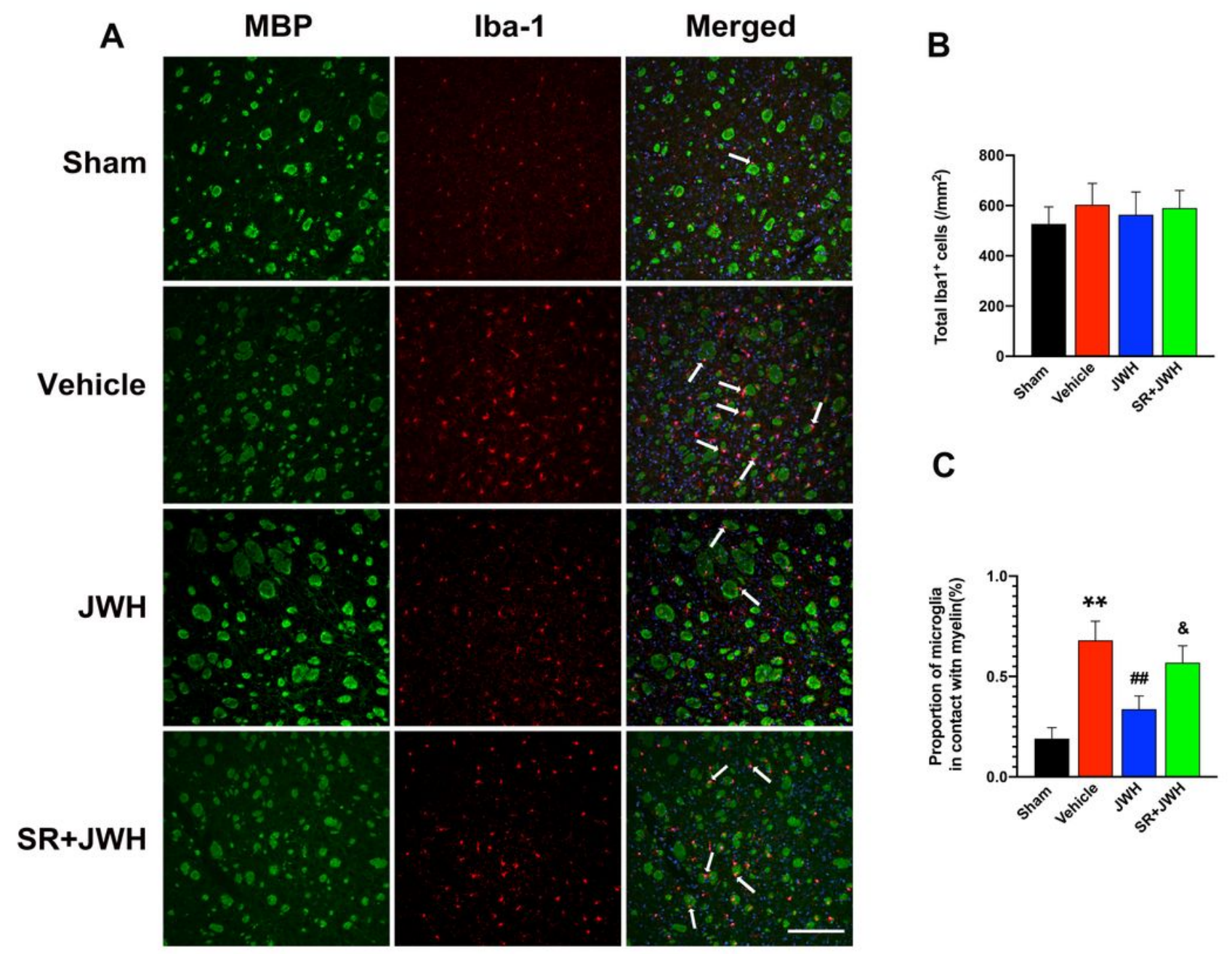

Figure 7

A CB2 agonist regulates microglia redistribution in the striatum in the context of TBI. Treatment with JWH133 did not influence the total number of microglia (A, B), but reduced the proportion of microglia in contact with myelin fibers at $3 \mathrm{~d}$ after TBI (A, C, indicated by white arrows). Scale bar $=20 \mu \mathrm{m}$. The data are expressed as the means $\pm S D s, n=6$. ${ }^{*} P<0.01$ compared to the sham group, \#\#P<0.01 compared to the vehicle group, and \&P $<0.05$ compared to the JWH group. 

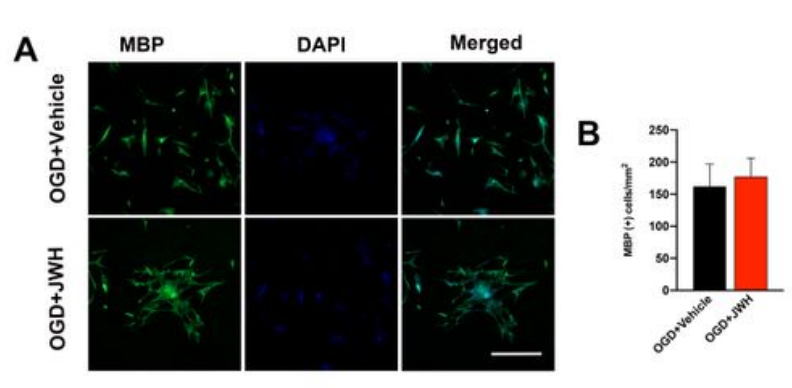

E
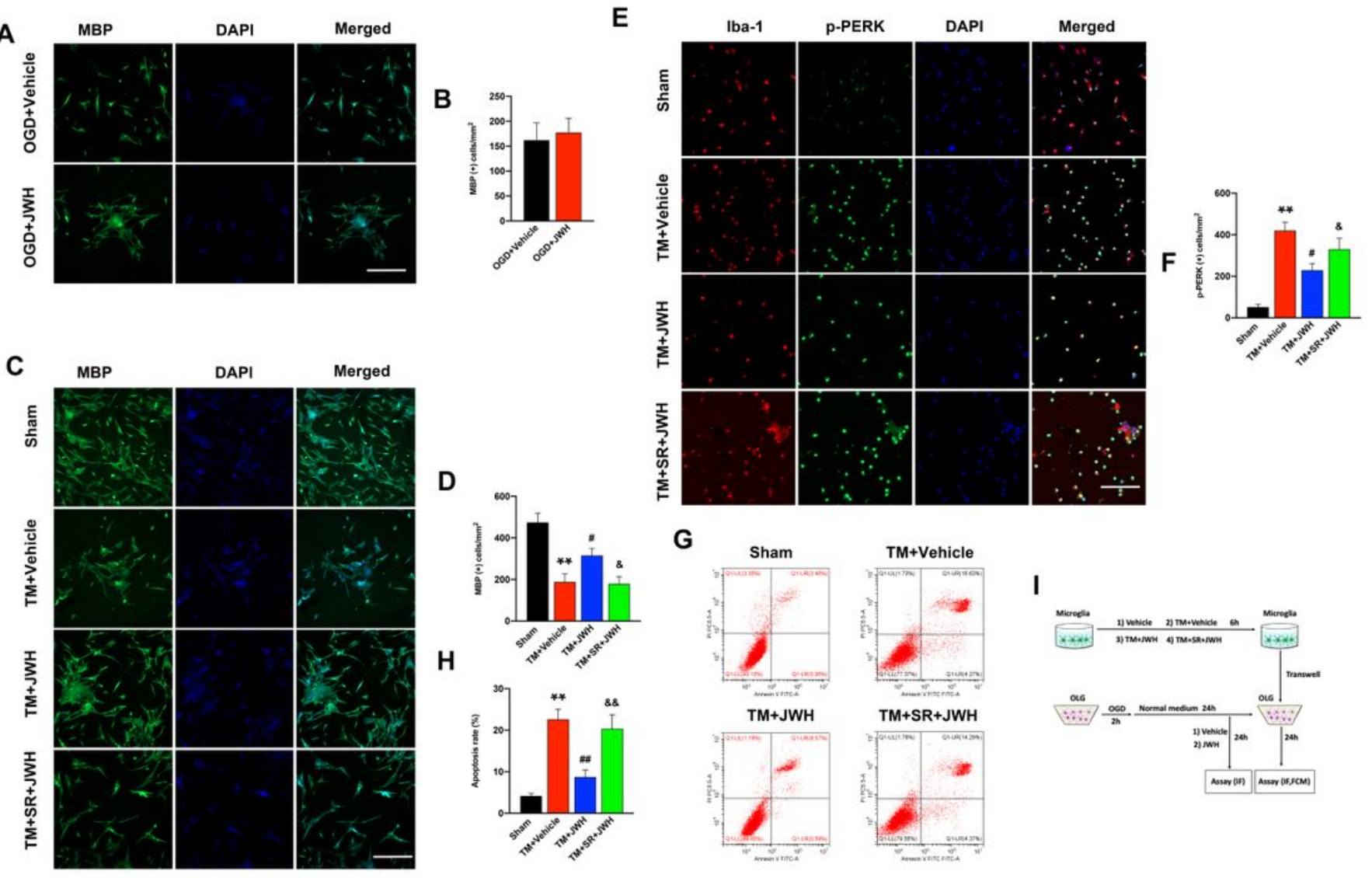

G
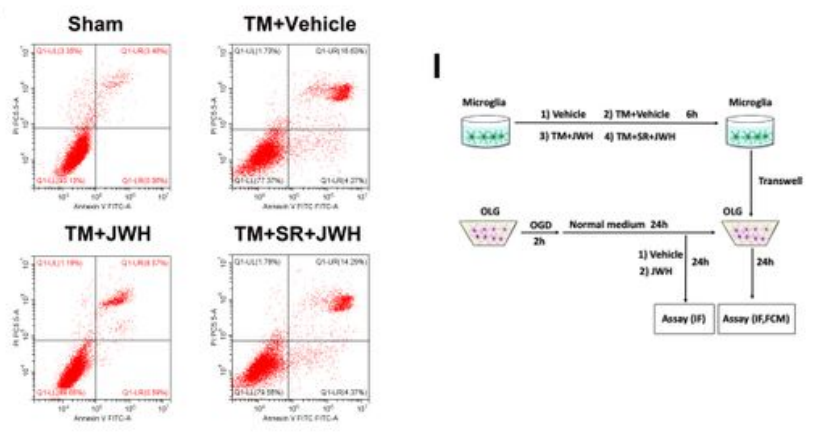

\section{Figure 8}

A CB2 agonist indirectly reduces OL injury via microglia through the PERK pathway in vitro. $(A, B)$ JWH133 provided little direct protection of primary OLs against OGD (P>0.05). (C, D, G, H) JWH133 protected OLs and reduced the OL apoptosis rate indirectly through microglia in in vitro experiments using a transwell system. (E, F) JWH133 prevented the increases in p-PERK levels induced by tunicamycin in primary microglia. (I) In vitro experiments were performed using a transwell system. Primary microglia were incubated with vehicle, tunicamycin $(3 \mu \mathrm{g} / \mathrm{ml})$, tunicamycin $(3 \mu \mathrm{g} / \mathrm{ml})+\mathrm{JWH} 133$ $(4 \mu \mathrm{M})$ or tunicamycin $(3 \mu \mathrm{g} / \mathrm{ml})+\operatorname{SR} 144528(1 \mu \mathrm{M})+\mathrm{JWH} 133(4 \mu \mathrm{M})$ for $6 \mathrm{~h}$. Cultured OLs were exposed to $2 \mathrm{~h}$ of OGD and returned to normal medium. Twenty-four hours later, the OLs were cocultured with primary microglia in a transwell system for $24 \mathrm{~h}$. The data are expressed as the means $\pm S D s, n=6$. ${ }^{*} \mathrm{P}<0.05$ and ${ }^{*} \mathrm{P}<0.01$ compared to the sham group, \#P $<0.05$ and \#\#P 0.01 compared to the vehicle group, and \&P $<0.05$ and \&\&P $<0.01$ compared to the JWH group. Scale bars=20 $\mu \mathrm{m}$. OLG, OLs; TM, tunicamycin. 


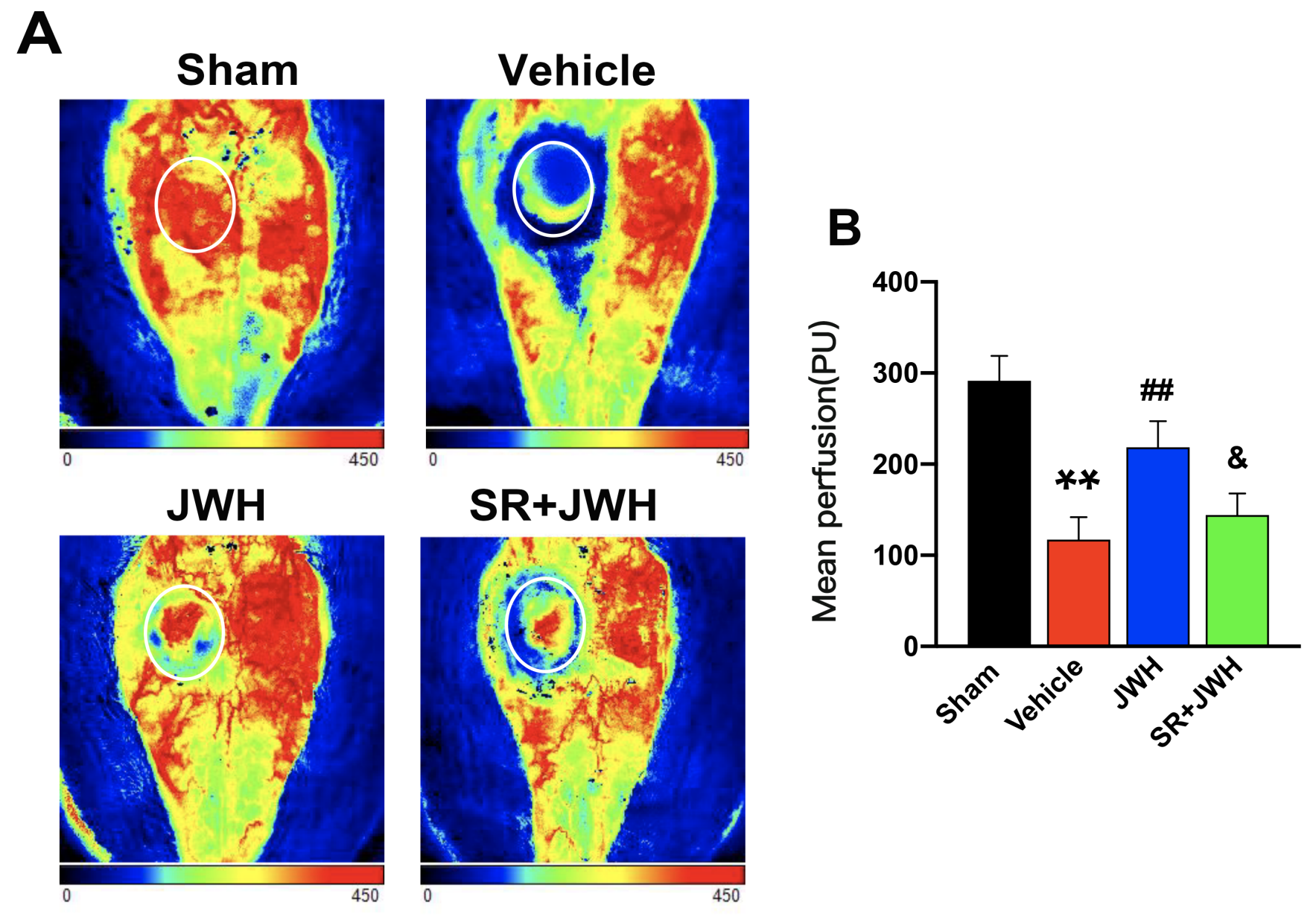

Figure 9

A CB2 agonist increases cerebral blood flow after TBI. (A, B) Treatment with JWH133 promoted cerebral blood flow recovery at $3 \mathrm{~d}$ following TBI. The data are expressed as the means $\pm S D s, n=6$. ${ }^{*} P<0.01$ compared to the sham group, \#\#P $<0.01$ compared to the vehicle group, and \&P $<0.05$ compared to the JWH group. 
A
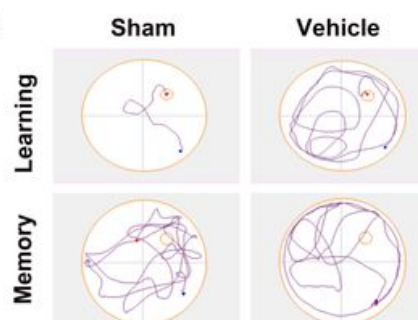

JWH

SR+JWH

H

Sham

Vehicle
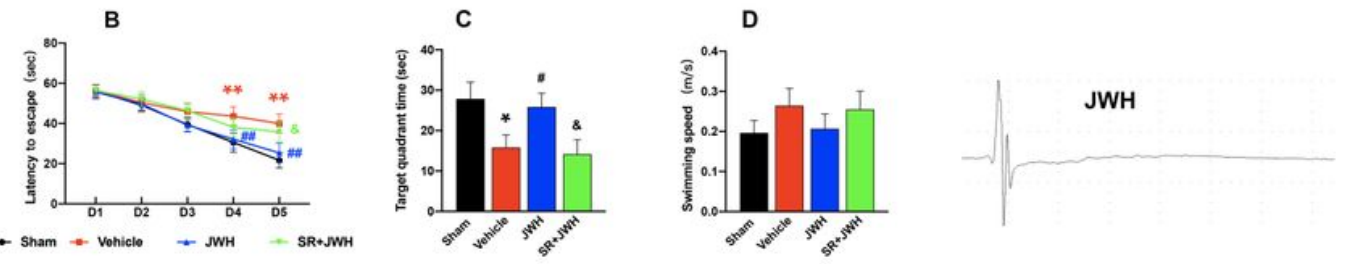

SR+JWH
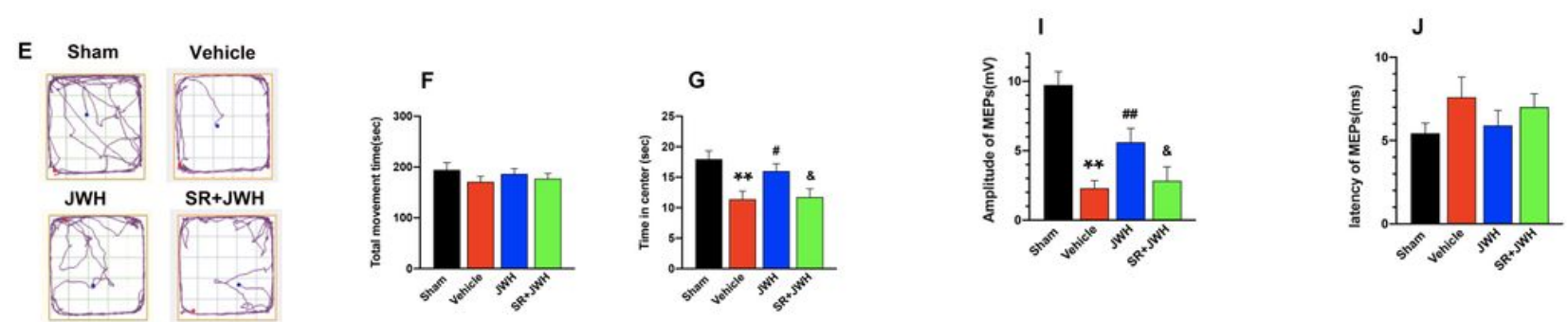

Figure 10

A CB2 agonist promotes restoration of neurological impairments and improves anxiety and depressivelike disorder. (A-D) JWH133 enhanced recovery of learning and memory deficits, as assessed with a Morris water maze at $14 \mathrm{~d}$ after TBI. (H-J) JWH133 enhanced recovery of motor function as indicated by MEPs at $14 \mathrm{~d}$ post injury. Further quantitative analysis indicated that JWH133 mainly affected the MEP amplitudes (I) but not MEP latencies ( $\mathrm{J}, \mathrm{P}>0.05)$. TBI rats treated with JWH133 showed similar movement times $(E, F)$; however, the treated animals spent significantly greater percentages of the time in the center $(E, G)$. The data are expressed as the means $\pm S D s, n=6 .{ }^{*} P<0.05$ and $* * P<0.01$ compared to the sham group, \#P $<0.05$ and \#\#P $<0.01$ compared to the vehicle group, and \&P $<0.05$ and $\& \& P<0.01$ compared to the JWH group.

\section{Supplementary Files}

This is a list of supplementary files associated with this preprint. Click to download.

- equations.docx 